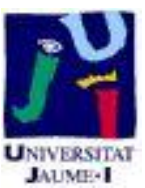

Título artículo / Títol article: Restructuring the Tridiagonal and Bidiagonal QR Algorithms for Performance

Autores / Autors

Fiel G. Van Zee, Robert A. Van de Geijn, Gregorio Quintana Ortí

Revista:

ACM Transactions on Mathematical Software, v. 40, issue 3 (April 2014)

Versión / Versió:

Postprint de l'autor

Cita bibliográfica / Cita bibliogràfica (ISO 690):
VAN ZEE, F. G.; VAN DE GEIJN, R. A.;

QUINTANA ORTÍ, G. Restructuring the Tridiagonal and Bidiagonal QR Algorithms for Performance.

ACM Transactions on Mathematical Software, v. 40, issue 3 (April 2014) , pp. 18:2-18:34

url Repositori UJI:

http://repositori.uji.es/xmlui/handle/10234/123806 


\title{
Restructuring the Tridiagonal and Bidiagonal QR Algorithms for Performance
}

FIELD G. VAN ZEE, The University of Texas at Austin

ROBERT A. VAN DE GEIJN, The University of Texas at Austin

GREGORIO QUINTANA-ORTí, Universitat Jaume I

\begin{abstract}
We show how both the tridiagonal and bidiagonal QR algorithms can be restructured so that they become rich in operations that can achieve near-peak performance on a modern processor. The key is a novel, cache-friendly algorithm for applying multiple sets of Givens rotations to the eigenvector/singular vector matrix. This algorithm is then implemented with optimizations that (1) leverage vector instruction units to increase floating-point throughput, and (2) fuse multiple rotations to decrease the total number of memory operations. We demonstrate the merits of these new QR algorithms for computing the Hermitian eigenvalue decomposition (EVD) and singular value decomposition (SVD) of dense matrices when all eigenvectors/singular vectors are computed. The approach yields vastly improved performance relative to the traditional QR algorithms for these problems and is competitive with two commonly used alternativesCuppen's Divide and Conquer algorithm and the Method of Multiple Relatively Robust Representationswhile inheriting the more modest $\mathcal{O}(n)$ workspace requirements of the original QR algorithms. Since the computations performed by the restructured algorithms remain essentially identical to those performed by the original methods, robust numerical properties are preserved.
\end{abstract}

Categories and Subject Descriptors: G.4 [Mathematical Software]: Efficiency

General Terms: Algorithms; Performance

Additional Key Words and Phrases: eigenvalues, singular values, tridiagonal, bidiagonal, EVD, SVD, QR algorithm, Givens rotations, linear algebra, libraries, high-performance

\section{INTRODUCTION}

The tridiagonal (and/or bidiagonal) QR algorithm is taught in a typical graduate-level numerical linear algebra course, and despite being among the most accurate ${ }^{1}$ methods for performing eigenvalue and singular value decompositions (EVD and SVD, respectively), it is not used much in practice because its performance is not competitive [Watkins 1982; Golub and Loan 1996; Stewart 2001; Dhillon and Parlett 2003]. The reason for this is twofold: First, classic QR algorithm implementations, such as those in LAPACK, cast most of their computation (the application of Givens rotations) in terms of a routine that is absent from the BLAS, and thus is typically not available in optimized form. Second, even if such an optimized implementation existed, it would

${ }^{1}$ Notable algorithms which exceed the accuracy of the QR algorithm include the $d q d s$ algorithm (a variant of the QR algorithm) [Fernando and Parlett 1994; Parlett and Marques 1999] and the Jacobi-SVD algorithm by [Drmač and Veselić 2008a; 2008b].

Authors' addresses: Field G. Van Zee and Robert A. van de Geijn, Institute for Computational Engineering and Sciences, Department of Computer Science, The University of Texas at Austin, Austin, TX 78712 , \{field, rvdg\}@cs . utexas . edu. Gregorio Quintana-Ortí, Departamento de Ingeniería y Ciencia de Computadores, Universitat Jaume I, Campus Riu Sec, 12.071, Castellón, Spain, gquintan@icc.uji.es.

Permission to make digital or hard copies of part or all of this work for personal or classroom use is granted without fee provided that copies are not made or distributed for profit or commercial advantage and that copies show this notice on the first page or initial screen of a display along with the full citation. Copyrights for components of this work owned by others than ACM must be honored. Abstracting with credit is permitted. To copy otherwise, to republish, to post on servers, to redistribute to lists, or to use any component of this work in other works requires prior specific permission and/or a fee. Permissions may be requested from Publications Dept., ACM, Inc., 2 Penn Plaza, Suite 701, New York, NY 10121-0701 USA, fax +1 (212) 869-0481, or permissions@acm.org.

(C) 2012 ACM 0098-3500/2012/01-ART00 $\$ 10.00$

DOI 10.1145/0000000.0000000 http://doi.acm.org/10.1145/0000000.0000000 
not matter because the $\mathrm{QR}$ algorithm is currently structured to apply Givens rotations via repeated instances of $\mathcal{O}\left(n^{2}\right)$ computation on $\mathcal{O}\left(n^{2}\right)$ data, effectively making it rich in level-2 BLAS-like operations, which inherently cannot achieve high performance because there is little opportunity for reuse of cached data. Many in the numerical linear algebra community have long speculated that the $\mathrm{QR}$ algorithm's performance could be improved by saving up many sets of Givens rotations before applying them to the matrix in which eigenvectors or singular vectors are being accumulated. In this paper, we show that, when computing all eigenvectors of a dense Hermitian matrix or all singular vectors of a dense general matrix, dramatic improvements in performance can indeed be achieved.

This work makes a number of contributions to this subject:

- It describes how the traditional QR algorithm can be restructured so that computation is cast in terms of an operation that applies many sets of Givens rotations to the matrix in which the eigen-/singular vectors are accumulated. This restructuring preserves a key feature of the original $\mathrm{QR}$ algorithm: namely, the approach requires only linear $(\mathcal{O}(n))$ workspace. An optional optimization to the restructured algorithm that requires $\mathcal{O}\left(n^{2}\right)$ workspace is also discussed and tested.

- It proposes an algorithm for applying many sets of Givens rotations that, in theory, exhibits greatly improved reuse of data in the cache. It then shows that an implementation of this algorithm can achieve near-peak performance by (1) utilizing vector instruction units to increase floating-point operation throughput, and (2) fusing multiple rotations so that data can be reused in-register, which decreases costly memory operations.

- It exposes and leverages the fact that lower computational costs of both the method of Multiple Relatively Robust Representations (MRRR) [Dhillon and Parlett 2004; Dhillon et al. 2006] and Cuppen's Divide-and-Conquer method (D\&C) [Cuppen 1980] are partially offset by an $\mathcal{O}\left(n^{3}\right)$ difference in cost between the former methods' backtransformations and the corresponding step in the $\mathrm{QR}$ algorithm.

- It demonstrates performance of EVD via the QR algorithm that is competitive with that of D\&C- and MRRR-based EVD, and QR-based SVD performance that is comparable to D\&C-based SVD.

- It makes the resulting implementations available as part of the open source libflame library.

The paper primarily focuses on the complex case; the mathematics then trivially simplify to the real case.

We consider these results to be significant in part because we place a premium on simplicity and reliability. The restructured QR algorithm presented in this paper is simple, gives performance that is almost as good as that of more intricate algorithms (such as D\&C and MRRR) and does so using only $\mathcal{O}(n)$ workspace, and without the worry of what might happen to numerical accuracy in pathological cases such as tightly clustered eigen-/singular values.

It should be emphasized that the improved performance we report is not so pronounced that the QR algorithm becomes competitive with D\&C and MRRR when performing standalone tridiagonal EVD or bidiagonal SVD (that is, when the input matrix is already reduced to condensed form). Rather, we show that in the context of the dense decompositions, which include two other stages of $\mathcal{O}\left(n^{3}\right)$ computation, the restructured $\mathrm{QR}$ algorithm provides enough speedup over the more traditional method to facilitate competitive overall performance. 


\section{COMPUTING THE SPECTRAL DECOMPOSITION OF A HERMITIAN MATRIX}

Given a Hermitian matrix $A \in \mathbb{C}^{n \times n}$, its eigenvalue decomposition (EVD) is given by $A=Q D Q^{H}$, where $Q \in \mathbb{C}^{n \times n}$ is unitary $\left(Q^{H} Q=I\right)$ and $D \in \mathbb{R}^{n \times n}$ is diagonal. The eigenvalues of matrix $A$ can then be found on the diagonal of $D$ while the corresponding eigenvectors are the columns of $Q$. The standard approach to computing the EVD proceeds in three steps [Stewart 2001]: Reduce matrix $A$ to real tridiagonal form $T$ via unitary similarity transformations: $A=Q_{A} T Q_{A}^{H}$; Compute the EVD of $T: T=Q_{T} D Q_{T}^{T}$; and back-transform the eigenvectors of $T: Q=Q_{A} Q_{T}$ so that $A=Q D Q^{H}$. Let us discuss these in more detail. Note that we will use the general term "workspace" to refer to any significant space needed beyond the $n \times n$ space that holds the input matrix $A$ and the $n$-length space that holds output eigenvalues (ie: the diagonal of $D$ ).

\subsection{Reduction to real tridiagonal form}

The reduction to tridiagonal form proceeds as the computation and application of a sequence of Householder transformations. When the transformations are defined as reflectors [Golub and Loan 1996; Van Zee et al. 2012; Stewart 2001], the tridiagonal reduction takes the form $H_{n-2} \cdots H_{1} H_{0} A H_{0} H_{1} \cdots H_{n-2}=Q_{A}^{H} A Q_{A}=T$, a real-valued, tridiagonal matrix. ${ }^{2}$

The cost of the reduction to tridiagonal form is $\frac{4}{3} n^{3}$ floating-point operations (flops) if $A$ is real and $4 \times \frac{4}{3} n^{3}$ flops if it is complex-valued. About half of those computations are in symmetric (or Hermitian) matrix-vector multiplications (a level-2 BLAS operation [Dongarra et al. 1988]), which are inherently slow since they perform $\mathcal{O}\left(n^{2}\right)$ computations on $\mathcal{O}\left(n^{2}\right)$ data. Most of the remaining flops are in Hermitian rank- $k$ updates (a level-3 BLAS operation) which can attain near-peak performance on modern architectures. If a flop that is executed as part of a level-2 BLAS operation is $K_{2}$ times slower than one executed as part of a level-3 BLAS operation, we will give the effective cost of this operation as $\left(K_{2}+1\right) \frac{2}{3} n^{3}$, where $K_{2} \geq 1$ and typically $K_{2} \gg 1$. Reduction to tridiagonal form requires approximately $b n$ workspace elements, where $b$ is the algorithmic blocksize used in the blocked algorithm [Van Zee et al. 2012].

\subsection{Spectral decomposition of $T$}

LAPACK [Anderson et al. 1999] provides four algorithms for computing the tridiagonal EVD (eigenvalues and eigenvectors), which we now discuss.

The $Q R$ algorithm $(Q R)$. The cost of the $Q R$ algorithm is approximated by $3 f n^{3}$, where $f$ equals the average number of Francis steps before deflation when a trailing eigenvalue has been found. The rule of thumb is that $1 \leq f \leq 2$. For a complex matrix $A$, the cost becomes $2 \times 3 f n^{3}$ because updating a complex eigenvector matrix $Q_{A}$ with real Givens rotations requires twice as many flops.

The fundamental problem with traditional QR algorithm implementations, such as the one currently found in LAPACK, is that this $\mathcal{O}\left(n^{3}\right)$ computation is cast in terms of a level-2 BLAS-like operation [Dongarra et al. 1988], which itself is actually implemented in terms of a level-1 BLAS-like operation [Lawson et al. 1979]. Such an implementation inherently cannot achieve high performance. If we assume that such operations are $K_{1}$ slower than those performed as part of a level-3 BLAS operation, the

\footnotetext{
${ }^{2}$ Actually, this produces a matrix with complex off-diagonal elements, which are then "fixed" to become real, as described in [Van Zee et al. 2012; Stewart 2001], an unimportant detail for the present discussion. Note that LAPACK does not need to fix the off-diagonal elements because the library defines Householder transformations in such a way that $T$ is reduced directly to real tridiagonal form. However, the cost of this minor optimization is that the transformations do not retain the property of being reflectors (ie: $H H \neq I$ ), and thus they must sometimes be conjugate-transposed depending on the direction from which they are applied.
} 
effective cost becomes $3 K_{1} f n^{3}$. Generally, $K_{1} \geq K_{2} \gg 1$. The main contribution of this paper is the restructuring of the computation so that the cost approaches $(2 \times) 3 \mathrm{fn}^{3}$.

The QR algorithm typically requires only $2(n-1)$ real-valued elements of workspace, which constitutes the maximum needed to hold the scalars which define the Givens rotations of any single Francis step. The eigenvectors overwrite the original matrix $A$.

Cuppen's Divide and Conquer $(D \& C)$. An implementation of $\mathrm{D} \& \mathrm{C}$, similar to the one in LAPACK, costs approximately $\frac{4}{3} \alpha^{2} n^{3}\left(1-4^{-t}\right)$ flops, where $t=\log _{2} n-1$ and $\alpha=1-\delta$ [Rutter 1994]. Here $\delta$ represents a typical value for the fraction of eigenvalues at any given level of recursion that deflate. Notice that $\left(1-4^{-t}\right)$ quickly approaches 1 as $n$ grows larger, and thus we drop this term in our analysis. It has been shown that the D\&C algorithm is, in practice, quite fast when operating on matrices that engender a high number of deflations [Cuppen 1980; Rutter 1994; Gu and Eisenstat 1995b]. This is captured in the $\alpha$ parameter, which attenuates quadratically as the number of deflations increase. A small value for $\alpha$ can significantly lessen the impact of the $n^{3}$ term. However, for some types of matrices, the number of deflations is relatively low, in which case the algorithm behaves more like a typical $\mathcal{O}\left(n^{3}\right)$ algorithm.

The LAPACK implementation of D\&C requires $n^{2}$ complex-valued elements and $2 n^{2}+4 n$ real-valued elements of workspace to form and compute with $Q_{T}$, in additional to $5 n$ additional integer elements.

Bisection and Inverse Iteration (BII). Bisection and Inverse Iteration (BII) requires $\mathcal{O}\left(n^{2}\right)$ operations to compute eigenvalues and, in the best case, another $\mathcal{O}\left(n^{2}\right)$ operations to compute the corresponding eigenvectors [Parlett 1980; Demmel et al. 1995; Demmel et al. 2008]. For matrices with clustered eigenvalues, the latter cost rises to $\mathcal{O}\left(n^{3}\right)$ as the algorithm must also perform modified Gram-Schmidt orthogonalization in an attempt to produce orthogonal eigenvectors [Demmel et al. 2008; Dhillon 1998]. This method of reorthogonalization, however, has been shown to cause BII to fail in both theory and practice [Dhillon 1998]. Thus, its use in the community has waned.

The implementation of BII in LAPACK requires $n^{2}, 5 n$, and $5 n$ complex-, real-, and integer-valued elements of workspace, respectively.

Multiple Relatively Robust Representations (MRRR). MRRR is a more recent algorithm that guarantees $\mathcal{O}\left(n^{2}\right)$ computation even in the worst case: when the matrix contains a cluster of eigenvalues, where each eigenvalue in the cluster is identical to $d$ digits of precision, the algorithm must perform work proportional to $d n^{2}$ [Demmel et al. 2008].

The MRRR algorithm is widely reported to require only linear $\mathcal{O}(n)$ workspace [Anderson et al. 1999; Dhillon et al. 2006; Demmel et al. 2008]. But in the context of a dense Hermitian eigenproblem, the workspace required is actually $n^{2}$ realvalued elements for the same reason that the D\&C and BII algorithms require $\mathcal{O}\left(n^{2}\right)$ workspace-namely, the algorithm inherently must explicitly compute and store the eigenvectors of $T$ before applying $Q_{A}$ towards the formation of $Q$. The latest implementation of MRRR in LAPACK as of this writing (version 3.3.1) requires $n^{2}+22 n$ and $10 n$ real- and integer-valued elements of workspace, respectively.

\subsection{Back-transformation}

D\&C, BII, and MRRR all share the property that they compute and store $Q_{T}$ explicitly as a dense matrix. The matrix $Q_{A}$ is stored implicitly as Householder transformations, which are applied to $Q_{T}$, yielding $Q$. This step is known as the backtransformation. The application of Householder transforms can be formulated as a blocked algorithm (either with the traditional compact WY-transform [Bischof and van Loan 1987; Schreiber and van Loan 1989] or with the UT-transform used by libflame 


\begin{tabular}{|l|c|c|c|}
\hline \multirow{2}{*}{ EVD Algorithm } & \multicolumn{3}{|c|}{ Effective higher-order floating-point operation cost } \\
\cline { 2 - 4 } & $A \rightarrow Q_{A} T Q_{A}^{H}$ & $T \rightarrow Q_{T} D Q_{T}^{T}$ & Form/Apply $Q_{A}$ \\
\hline original QR & $4 \times\left(K_{2}+1\right) \frac{2}{3} n^{3}$ & $2 \times K_{1} 3 f n^{3}$ & $4 \times \frac{4}{3} n^{3}$ \\
\hline restructured QR & $4 \times\left(K_{2}+1\right) \frac{2}{3} n^{3}$ & $2 \times 3 f n^{3}$ & $4 \times \frac{4}{3} n^{3}$ \\
\hline D\&C & $4 \times\left(K_{2}+1\right) \frac{2}{3} n^{3}$ & $\frac{4}{3} \alpha^{2} n^{3}$ & $4 \times 2 n^{3}$ \\
\hline BII & $4 \times\left(K_{2}+1\right) \frac{2}{3} n^{3}$ & $\mathcal{O}\left(n^{2}\right) \sim \mathcal{O}\left(n^{3}\right)$ & $4 \times 2 n^{3}$ \\
\hline MRRR & $4 \times\left(K_{2}+1\right) \frac{2}{3} n^{3}$ & $\mathcal{O}\left(n^{2}\right)$ & $4 \times 2 n^{3}$ \\
\hline
\end{tabular}

Fig. 1. A summary of approximate floating-point operation cost for suboperations of various algorithms for computing a Hermitian eigenvalue decomposition. Integer scalars at the front of the expressions (e.g. " $4 \times \ldots$..) indicate the floating-point multiple due to performing arithmetic with complex values. In the cost estimates for the $\mathrm{QR}$ algorithms, $f$ represents the typical number of Francis steps needed for an eigenvalue to converge. For Cuppen's Divide-and-conquer algorithm, $\alpha$ represents the number of non-deflations in each recursive subproblem.

[Joffrain et al. 2006; Van Zee et al. 2012]) at a cost of approximately $2 n^{3}$ flops (multiplied by four for complex). This casts most computation in terms of level-3 BLAS.

When the $\mathrm{QR}$ algorithm is employed, $Q_{A}$ is formed before the $\mathrm{QR}$ algorithm is called. Forming $Q_{A}$ is done "in place," overwriting matrix $A$ at a cost of approximately $\frac{4}{3} n^{3}$ flops (times four for complex), cast mostly in terms of level-3 BLAS. The Givens rotations are subsequently applied directly to $Q_{A}$. Thus, the back-transformations of D\&C and MRRR incur an additional $\frac{2}{3} n^{3}$ flops (times four for complex) relative to the corresponding stage of the $\mathrm{QR}$ algorithm. Later, we will show that this additional $\mathcal{O}\left(n^{3}\right)$ term in the cost of EVD via D\&C or MRRR will partially offset the cost savings of performing the tridiagonal EVD stage with either of these aforementioned methods.

\subsection{Numerical accuracy}

A recent paper evaluated the numerical accuracy of the four tridiagonal eigenvalue algorithms available in LAPACK [Demmel et al. 2008]. The authors observed that, in practice, the $\mathrm{QR}$ and $\mathrm{D} \& \mathrm{C}$ algorithms were the most accurate, exhibiting $\mathcal{O}(\sqrt{n} \varepsilon)$ accuracy, where $n$ is the dimension of the matrix and $\varepsilon$ is the machine precision. ${ }^{3}$ By contrast, the accuracy of BII and MRRR was observed to be approximately $\mathcal{O}(n \varepsilon)$. Another paper compared the numerical performance of the netlib MRRR (as of LAPACK 3.3.2), netlib D\&C, and an improved MRRR algorithm. The authors found that netlib MRRR produced unsatisfactory results for "not too few" of the test cases [Willems and Lang 2011]. The authors assert that the "best method for computing all eigenvalues" is a derivative of the $\mathrm{QR}$ algorithm, known as the $d q d s$ algorithm [Fernando and Parlett 1994; Parlett and Marques 1999], but suggest that it is avoided only because it is, in practice, found to be "very slow."

Recent efforts at producing accurate SVD algorithms have focused on Jacobi iteration [Drmač and Veselić 2008a; 2008b; Drmač 2009]. Admittedly, these algorithms tend to be somewhat more accurate than the QR algorithm [Demmel and Veselić 1992].

\footnotetext{
${ }^{3}$ This paper investigated accuracy only for tridiagonal matrices. By contrast, our work concerns the dense problem, which also includes a tridiagonal/bidiagonal reduction pre-process as well as a back-transformation step. Since these steps are implemented in terms of Householder transformations, we believe them to be quite accurate, but examining their precise numerical impact on the multi-stage EVD or SVD is beyond the scope of this paper.

${ }^{4}$ Note that if the matrix generated by the application is already tridiagonal, then D\&C and MRRR would be the methods of choice. If, additionally, only a few eigenvectors are desired and the application can accept the possibility of somewhat less accurate results, then MRRR may be preferred.
} 


\begin{tabular}{|c|c|c|c|}
\hline \multirow{2}{*}{ EVD Algorithm } & \multicolumn{3}{|c|}{ Workspace required for optimal performance } \\
\hline & $A \rightarrow Q_{A} T Q_{A}^{H}$ & $T \rightarrow Q_{T} D Q_{T}^{T}$ & Form/Apply $Q_{A}$ \\
\hline original QR & $\begin{array}{l}\mathbb{C}:(b+1) n \\
\mathbb{R}: 2 n-1\end{array}$ & $\mathbb{R}: 2(n-1)$ & $\mathbb{C}: b(n-1)$ \\
\hline restructured $\mathrm{QR}$ & $\begin{array}{l}\mathbb{C}:(2 b+1) n \\
\mathbb{R}: 2 n-1\end{array}$ & $\mathbb{R}: 2 b(n-1)$ & $\mathbb{C}: b(n-1)$ \\
\hline $\mathrm{D \& C}$ & $\begin{array}{l}\mathbb{C}:(b+1) n \\
\mathbb{R}: 2 n-1\end{array}$ & $\begin{array}{l}\mathbb{C}: n^{2} \\
\mathbb{R}: 2 n^{2}+4 n \\
\mathbb{Z}: 5 n\end{array}$ & $\mathbb{C}: b n$ \\
\hline BII & $\begin{array}{l}\mathbb{C}:(b+1) n \\
\mathbb{R}: 2 n-1\end{array}$ & $\begin{array}{l}\mathbb{C}: n^{2} \\
\mathbb{R}: 5 n \\
\mathbb{Z}: 5 n\end{array}$ & $\mathbb{C}: b n$ \\
\hline MRRR & $\begin{array}{l}\mathbb{C}:(b+1) n \\
\mathbb{R}: 2 n-1\end{array}$ & $\begin{array}{l}\mathbb{C}: n^{2} \\
\mathbb{R}: 20 n \\
\mathbb{Z}: 12 n\end{array}$ & $\mathbb{C}: b n$ \\
\hline
\end{tabular}

Fig. 2. A summary of workspace required to attain optimal performance for each suboperation of various algorithms employed in computing a Hermitian eigenvalue decomposition. Expressions denoted by $\mathbb{R}, \mathbb{C}$, and $\mathbb{Z}$ refer to the amount of real, complex, and integer workspace required. Some suboperations, such as forming or applying $Q$, may be executed with less workspace than indicated, but at a cost of significantly lower performance. Note that we consider workspace for the overall Hermitian EVD, not the individual stages in isolation. Thus, workspace refers to any storage needed beyond the $n \times n$ input matrix and the $n$-length vector needed to hold the eigenvalues.

However, Jacobi-based algorithms suffer from performance hurdles similar to that of conventional $\mathrm{QR}$ algorithms due to their common heavy reliance upon Givens rotations.

It goes without saying that some applications are dependent upon very high accuracy, even if these algorithms are slower for many classes of matrices. Therefore, realizing a $\mathrm{QR}$ algorithm that is restructured for high-performance becomes even more consequential.

\subsection{Cost comparison}

The cost and workspace requirements for each step of the EVD are summarized in Figures 1 and 2. We use these estimates to predict the benefits of a hypothetical restructured QR algorithm that applies Givens rotations with level-3 BLAS performance, shown in Figure 3. For that figure we make the following assumptions: First, we assume that $K_{1} \approx K_{2}$; Furthermore, since microprocessor cores are increasing in speed at a faster rate than memory architectures, and since level-1 and level-2 BLAS operations are memory-bound, $K_{1}$ and $K_{2}$ for future architectures will likely increase; the average number of Francis steps before deflation is taken as $f=1.5$ and $\alpha=0.5$ for $\mathrm{D} \& \mathrm{C}$; And we entirely ignore the $\mathcal{O}\left(n^{2}\right) \operatorname{cost}$ of $T \rightarrow Q_{T} D Q_{T}^{T}$ in the MRRR-based algorithm. We omit BII from our results because (1) in the best case, its performance is similar to that of MRRR, and (2) it has been shown to be numerically unreliable [Dhillon 1998].

Inspecting Figure 3, together with the cost expressions from Figure 1, we make the following observations:

- A restructured $Q R$ algorithm allows competitive EVD performance relative to MRRR. For instance, when $K_{1}=K_{2}=8$, an EVD using a restructured QR algorithm has the 


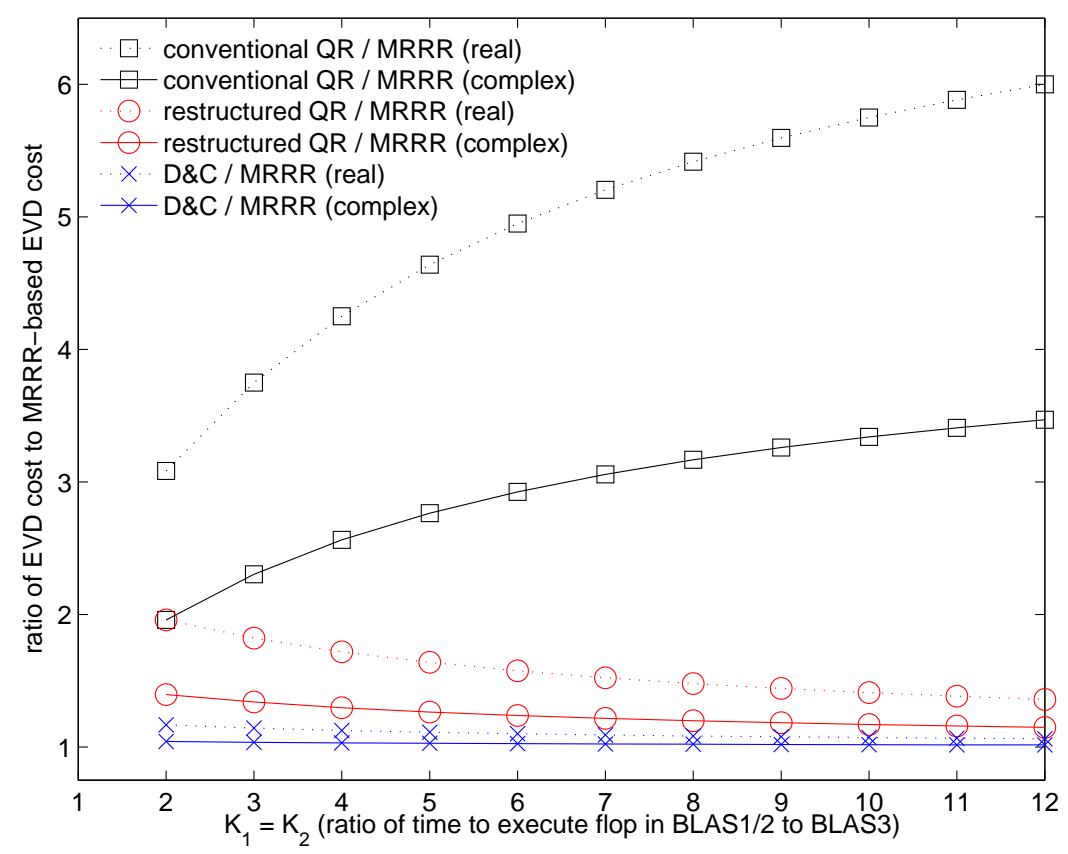

Fig. 3. Predicted ratios of floating-point operation cost for various Hermitian EVD algorithms relative to the cost of Hermitian EVD via MRRR. Note that these cost estimates include estimated costs of reduction to tridiagonal form (via Householder transformations) and also the corresponding back-transformation step.

potential to be only $48 \%$ and $20 \%$ slower than MRRR-based EVD for real and complex matrices, respectively. (In comparison, using the conventional QR algorithm results in EVD that is $442 \%$ and $217 \%$ slower for real and complex matrices, respectively.)

- As $K_{1}$ increases, the relative benefit of restructuring over a conventional QR algorithm quickly becomes significant.

- As $K_{2}$ increases, the reduction to tridiagonal form consumes a higher fraction of the total EVD cost, regardless of which method is used. This allows the restructured QR algorithm to become more competitive with D\&C- and MRRR-based EVD.

- While a restructured QR algorithm would still greatly benefit EVD on real matrices, it is the complex case where we would expect the algorithm to be most competitive with D\&C- and MRRR-based methods.

Thus, a high-performance QR algorithm is theoretically within reach. The question now becomes how to achieve this high performance.

\section{TRIDIAGONAL QR ALGORITHM BASICS}

We quickly review the basic ideas behind the (tridiagonal) QR algorithm for computing the EVD.

\subsection{Shifted tridiagonal QR algorithm}

Given a tridiagonal matrix $T$, the shifted $\mathrm{QR}$ algorithm is given by 


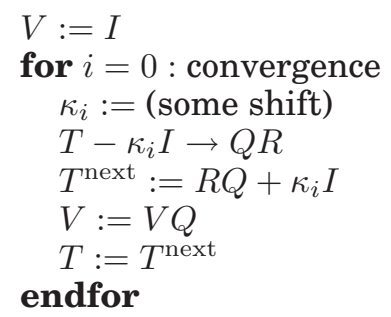

Here, $T \rightarrow Q R$ denotes a $\mathrm{QR}$ factorization. Under mild conditions this converges to a matrix of structure $\left(\begin{array}{c|c}T_{T L} & 0 \\ \hline 0 & \lambda\end{array}\right)$, where $\lambda$ is an eigenvalue of $T$. The process can then continue by deflating the problem, which means continuing the iteration with the tridiagonal matrix $T_{T L}$. Eventually, $T$ becomes a diagonal matrix, with the eigenvalues along its diagonal and $V$ converges to a matrix with the corresponding eigenvectors (of the original matrix) as its columns.

Convergence occurs when one of the off-diagonal elements $\tau_{i+1, i}$ becomes negligible. Following Stewart [Stewart 2001], $\tau_{i+1, i}$ is considered to be negligible when $\left|\tau_{i+1, i}\right| \leq$ $\varepsilon \sqrt{\left|\tau_{i, i} \tau_{i+1, i+1}\right|}$. Most often, deflation will occur in element $\tau_{n-1, n-2}$.

Two choices for shift $\kappa_{i}$ are commonly used: (1) The Rayleigh Quotient shift - This method consists of choosing $\kappa_{i}=\tau_{n-1, n-1}$ (i.e., the bottom-right-most element of the current tridiagonal matrix), yields cubic convergence [Stewart 2001], but has not been shown to be globally convergent; and (2) The Wilkinson shift — This shift chooses $\kappa_{i}$ as the eigenvalue of $\left(\begin{array}{cc}\tau_{n-2, n-2} & \tau_{n-1, n-2} \\ \tau_{n-1, n-2} & \tau_{n-1, n-1}\end{array}\right)$ that is closest to $\tau_{n-1, n-1}$, converges at least quadratically, and is globally convergent [Stewart 2001]. We will use the Wilkinson shift, which in practice appears to yield faster results.

\subsection{The Francis Implicit $Q$ Step}

One of the most remarkable discoveries in numerical linear algebra is the Francis Implicit Q Step, which provides a clever way of implementing a single iteration of the $\mathrm{QR}$ algorithm with a tridiagonal matrix [Watkins 1982]. Let $T-\kappa_{i} I$ equal

$$
T=\left(\begin{array}{cc|cccc}
\tau_{0,0}-\kappa_{i} & \tau_{1,0} & 0 & 0 & \cdots & 0 \\
\tau_{1,0} & \tau_{1,1}-\kappa_{i} & \tau_{1,2} & 0 & \cdots & 0 \\
\hline 0 & \tau_{2,1} & \tau_{2,2}-\kappa_{i} & \tau_{3,2} & \ddots & \vdots \\
0 & 0 & \tau_{3,2} & \ddots & \ddots & 0 \\
\vdots & \vdots & \ddots & \ddots & \tau_{n-2, n-2}-\kappa_{i} & \tau_{n-1, n-2} \\
0 & 0 & \cdots & 0 & \tau_{n-1, n-2} & \tau_{n-1, n-1}-\kappa_{i}
\end{array}\right)
$$

As part of the QR factorization of $T-\kappa_{i} I$, the first step is to annihilate $\tau_{1,0}$. For this purpose, we compute a Givens rotation $G_{0}^{T}=\left(\begin{array}{cc}\gamma_{0} & \sigma_{0} \\ -\sigma_{0} & \gamma_{0}\end{array}\right)$ that, when applied to the first two rows, annihilates $\tau_{1,0}$. (We label the rotations such that $G_{i}^{T}$ is the rotation that affects rows $i$ and $i+1$.) But rather than applying $G_{0}^{T}$ to $T-\kappa_{i} I$, we instead apply it to the original $T$, affecting the first two rows, after which we apply $G_{0}$ from the right 
to the first two columns. This yields a matrix of the form

$$
T=\left(\begin{array}{cc|cccc}
\tau_{0,0}^{\text {next }} & \star & \star & 0 & \cdots & 0 \\
\star & \star & \star & 0 & \cdots & 0 \\
\hline \star & \star & \tau_{2,2} & \tau_{3,2} & \ddots & \vdots \\
0 & 0 & \tau_{3,2} & \ddots & \ddots & 0 \\
\vdots & \vdots & \ddots & \ddots & \tau_{n-2, n-2} & \tau_{n-1, n-2} \\
0 & 0 & \cdots & 0 & \tau_{n-1, n-2} & \tau_{n-1, n-1}
\end{array}\right) .
$$

where the "^"s represent non-zeroes. This step of computing $G_{0}^{T}$ from $T-\kappa_{i} I$ and applying it to $T$ is said to "introduce the bulge" because it causes a non-zero entry to appear below the first subdiagonal (and, because of symmetry, above the first superdiagonal), which we have highlighted above. Next, we compute a rotation $G_{1}^{T}$ that will annihilate $\tau_{2,0}$ and apply it to the second two rows of the updated matrix, and its transpose to the second two columns. This begins the process of "chasing the bulge" and causes the non-zero entry to reappear at element $(3,1)$ :

$$
T=\left(\begin{array}{cc|cccc}
\tau_{0,0}^{\text {next }} & \tau_{1,0}^{\text {next }} & 0 & 0 & \cdots & 0 \\
\tau_{1,0}^{\text {next }} & \tau_{1,1}^{\text {next }} & \star & \star & \cdots & 0 \\
\hline 0 & \star & \star & \star & \ddots & \vdots \\
0 & \star & \star & \ddots & \ddots & 0 \\
\vdots & \vdots & \ddots & \ddots & \tau_{n-2, n-2} & \tau_{n-1, n-2} \\
0 & 0 & \cdots & 0 & \tau_{n-1, n-2} & \tau_{n-1, n-1}
\end{array}\right) .
$$

This process continues, computing and applying rotations $G_{0}, G_{1}, G_{2}, \ldots, G_{n-2}$ in this manner (transposed from the left, without transpose from the right) until the matrix is again tridiagonal. Remarkably, the $T$ that results from this process is, in theory, exactly the same as the $T^{\text {next }}$ in the original algorithm.

This process is known as a Francis Implicit Q Step (or, more concisely, a Francis step). Neither $Q$ nor $R$ nor $T$ is ever explicitly formed.

\subsection{Accumulating the eigenvectors}

If we initially let $V=I$, then $Q_{T}$ can be computed as follows. First, we partition $V$ into its columns: $V=\left(\begin{array}{llll}v_{0} & v_{1} & \cdots & v_{n-1}\end{array}\right)$. For each Francis step, for $j=0, \ldots, n-2$, apply the rotations $G_{j}$ to columns $v_{j}$ and $v_{j+1}$, in the order indicated by

$$
\begin{aligned}
& G_{0} \rightarrow G_{1} \rightarrow G_{2} \rightarrow G_{3} \rightarrow G_{4} \rightarrow G_{5} \rightarrow G_{6} \rightarrow G_{7} \rightarrow G_{8} \\
& \begin{array}{llllllllll}
v_{0} & v_{1} & v_{2} & v_{3} & v_{4} & v_{5} & v_{6} & v_{7} & v_{8} & v_{9}
\end{array}
\end{aligned}
$$

Here, the arrows indicate the order in which the Givens rotations must be applied while the two columns situated below each rotation represent the columns $v_{j}$ and $v_{j+1}$ to which that rotation is applied. We refer to $\left\{G_{0}, \ldots, G_{n-2}\right\}$ as a single Francis set of rotations. Following this process, after the $\mathrm{QR}$ iteration is complete and $T$ has converged to $D$ (i.e., the eigenvalues of $T$ ), $V=Q_{T}$. That is, column $v_{j}$ contains the eigenvector of $T$ that corresponds to the eigenvalue $\delta_{j, j}$. 


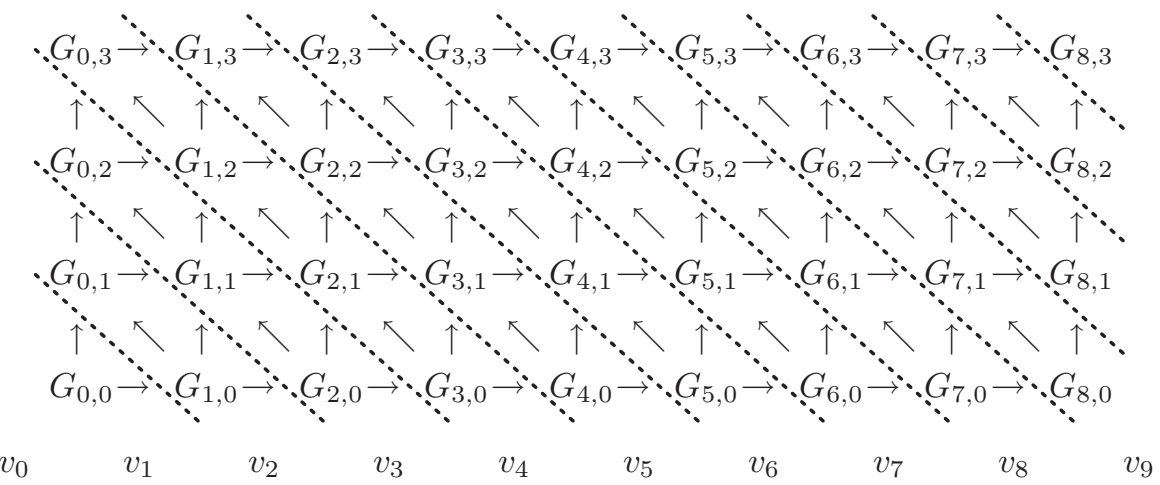

Fig. 4. Applying Givens rotations from four Francis steps to a matrix $V$ consisting of 10 columns. A rotation $G_{j, i}$ is generated during the $i$ th Francis step and is applied to columns $v_{j}$ and $v_{j+1}$. For any given rotation $G$, inbound arrows indicate dependencies that must be satisfied before the rotation may be performed. Temporal and spatial locality may be increased by applying rotations in diagonal "waves" from left to right (where the rotations in each wave are separated by dotted lines), and from bottom-right to top-left within each wave, according to the dependencies shown.

If we initialize $V:=Q_{A}$ and the rotations computed from each Francis step are applied to $V$ as previously described, then upon completion of the $\mathrm{QR}$ iteration $V=Q$, where column $v_{j}$ contains the eigenvector of $A$ associated with the eigenvalue $\delta_{j, j}$.

The application of a Givens rotation to a pair of columns of $Q$ requires approximately $6 n$ flops $(2 \times 6 n$ flops for complex $V)$. Thus, applying $n-1$ rotations to $n$ columns costs approximately $(2 \times) 6 n^{2}$ flops. Since performing the Francis step without applying to $Q$ takes $\mathcal{O}(n)$ computation, it is clearly the accumulation of eigenvectors that dominates the cost of the QR algorithm.

\subsection{LAPACK routines $\{$ sdcz $\}$ steqr}

The tridiagonal QR algorithm is implemented in LAPACK in the form of the subroutines ssteqr and dsteqr (for single- and double-precision real matrices, respectively) and csteqr and zsteqr (for single- and double-precision complex matrices, respectively). These routines implement the entire algorithm described in Sections 3.1-3.3 in a single routine. ${ }^{5}$ In these implementations, Givens rotations from a single Francis step are saved in temporary storage until the step is complete. These rotations, a Francis set, are then applied to $Q_{A}$ before moving on to the next Francis step. The application of the Givens rotations to the columns of $Q_{A}$ is implemented via calls to $\{\mathrm{sdcz}\}$ lasr, which provide flexible interfaces to routines that are very similar to the level-1 BLAS routines $\{$ sd $\}$ rot. This, inherently, means that LAPACK's $\{$ sdcz\}steqr routines do not benefit from the fast cache memories that have been part of modern microprocessors since the mid-1980s. Thus, accumulating eigenvectors is costly not only because of total number of flops, but also because those flops are performed at a very slow rate.

\section{APPLYING GIVENS ROTATIONS}

We now analyze the application of Givens rotations to a matrix. 


\subsection{The basic idea}

Recall from Section 3.3 that applying one Francis set of $(n-1)$ rotations to the $n \times n$ matrix $V$ in which eigenvectors are being accumulated requires approximately $6 n^{2}$ flops (or $2 \times 6 n^{2}$ for complex $V$ ). This means that $(2 \times) n^{2}$ floating-point numbers must be loaded from and stored back to main memory at least once each, for an absolute minimum number of $(2 \times) 2 n^{2}$ memory operations. 6 Thus, the ratio of flops to memops is at best 3 . In practice, this ratio is even lower. For instance, the LAPACK routines for performing this operation, $\{\mathrm{sdcz}\}$ lasr, perform $(2 \times) 6$ flops for every $(2 \times) 4 \mathrm{mem}$ ops, leaving them with a flop-to-memop ratio of just 1.5 . This is a problem given that a memop typically requires much more time to execute than a flop, and the ratio between them is getting worse as microprocessor speeds outpace memory technology. We conclude that updating the matrix one Francis set at a time will not be efficient.

The first key insight behind this paper is that if one instead applies $k$ Francis sets, the theoretical maximum number of flops per memop rises to $3 k$. Evidence that this opportunity exists is presented in Figure 4. In this example, there are $k=4$ Francis sets of rotations. The arrows indicate dependencies between rotations. The semantic meaning expressed by these arrows is simple: a rotation must have all dependencies (inbound arrows) satisfied before it may be performed. Notice that the drawing is a directed-acyclic graph (DAG) and hence a partial order. As long as the partial order is obeyed, the rotations are applied to columns in the exact same order as the traditional QR algorithm.

The second key insight is that the dotted lines in Figure 4 expose "waves" of rotations that may be applied. The idea here is that waves of rotations are applied from left to right with rotations applied from bottom-right to top-left within each wave. (Notice that this pattern of applying rotations obeys the partial order.) It is easy to see that this wave-based approach increases temporal and spatial data locality. In fact, once the wavefront is fully formed, only one new column of $V$ is brought into cache for each wave; all of the other columns needed for that wave are already residing in the cache.

The dependencies depicted in Figure 4 have been observed by others [Lang 1998; Kågström et al. 2008]. However, these efforts used the dependency graph to identify groups of waves which could be accumulated into an intermediate Givens rotation matrix and then applied to $Q_{A}$ via optimized level-3 BLAS operations dgemm and dtrmm. It is worth pointing out, though, that these two-stage implementations still need to accumulate individual Givens rotations into the intermediate rotations matrix. As a result, these efforts still stand to benefit from the insights in this paper because we will show how to accelerate the direct application of Givens rotations. In Section 6.4, we discuss an optional optimization to our restructured QR algorithm that, similar to the techniques used in [Lang 1998; Kågström et al. 2008], leverages dgemm. In that section, we compare and contrast the two approaches.

We are not the first to highlight the need for optimized routines for applying Givens rotations. As part of the BLAST forum [BLAST 2002] there were requests for a routine that would apply multiple sets of rotations to a matrix. However, a standard interface never materialized, and so library maintainers were never sufficiently motivated to include the operation in their BLAS libraries.

\subsection{Details of cache behavior}

Let us perform a more detailed (but still simple) analysis of the cache behavior of applying Givens rotations, as described in the previous section. Let us assume we have

\footnotetext{
${ }^{5}$ These routines, coded in Fortran-77, are likely direct translations from EISPACK, which dates back to the late 1960's. Evidence of this exists in the number of GO TO statements in the code.

${ }^{6}$ Henceforth, we will call the reading or writing of a floating-point number a memory operation, or memop.
} 
a fully-associative cache that uses the least-recently used replacement policy, and that the number of elements in our cache, $n_{C}$, is significantly less than $n^{2}$ but is greater than $k n$, where $k \ll n$. Also, let us ignore the fact that accessing data of unit stride often produces cache hits due to entire cache lines being loaded from memory. We justify ignoring this effect by pointing out that the scalar-level hit and miss pattern would be identical for all column-oriented algorithms and thus would not affect our analysis. The idea is that we wish to abstract away to hits and misses of entire columns of $V=Q_{A}$, given that applying Givens rotations inherently means touching entire columns of data. Thus, from now on, in the context of analyzing the application of Givens rotations, we will count only column-accesses, which refer to the memory operations needed to load or store $n$ consecutive elements. ${ }^{7}$

Let us begin by considering how we apply the first of $k$ Francis sets of Givens rotations using the single-step approach employed by the traditional QR algorithm. Applying the first set of rotations accesses most of the $n$ columns of $V$ twice, for a total of $2(n-1)$ column-accesses. Of these accesses, $n$ are cache misses and $n-2$ are cache hits. Now, we assume that since $n_{C} \ll n^{2}$, upon finishing applying the first Francis set and beginning the next, columns $v_{0}, v_{1}, v_{2}, \ldots$ have long since been evicted from the cache. Thus, there is no data reuse between sets of Givens rotations using the single-step approach found in LAPACK, resulting in $k n$ cache misses and $k(n-2)$ cache hits, for a hit rate of approximately $50 \%$.

We now highlight a simple observation. If multiple sets of rotations are accumulated, the order in which they are applied can be modified to improve the reuse of data in the caches. Consider the approach exemplified by Figure 4 . We identify three distinct "stages" of the wavefront algorithm. For our discussion, let us define these phases as follows:

- The startup phase refers to the initial $k-1$ waves of rotation applications where the "wavelength" (the number of rotations within the wave) is initially 1 and gradually increases.

- The pipeline phase refers to the middle $n-k$ waves of rotation applications where each wave is of length $k$.

- The shutdown phase refers to the final $k-1$ waves, where the wavelength becomes less than $k$ and gradually decreases to 1 .

Next, we analyze the cache behavior of this algorithm. The wavefront algorithm illustrated in Figure 4 produces $k \times 2(n-1)=2 k(n-1)$ column-accesses. Of these accesses, there are $2+(k-2)=k$ cache misses in the startup phase, $(n-1)-(k-1)=n-k$ cache misses in the pipeline phase, and no cache misses in the shutdown phase, for a total of $n$ cache misses. This means the ratio of cache misses to total accesses is approximately $\frac{n}{2 k(n-1)} \approx \frac{1}{2 k}$, and the fraction of cache hits is $1-\frac{1}{2 k}$. For larger values of $k$, this cache hit ratio is quite close to 1 , and as a result the wavefront algorithm should exhibit very favorable data access patterns.

\subsection{A wavefront algorithm}

We now give several algorithms for applying Givens rotations, leading up to an algorithm for wavefront application of $k$ sets of rotations to an $m \times n$ matrix. To our knowledge, this wavefront algorithm is previously unpublished. ${ }^{8}$

\footnotetext{
${ }^{7}$ If $n_{C}<n$, that is, if the matrix is so large that not even one column can fit in the cache, then we may employ the blocking technique discussed in Section 4.4, in which case the above analysis still holds.

${ }^{8}$ While we believe the wavefront algorithm for applying Givens rotations to be novel in the context of tridiagonal EVD via the QR algorithm, the idea of structuring computation in terms of successive waves is
} 


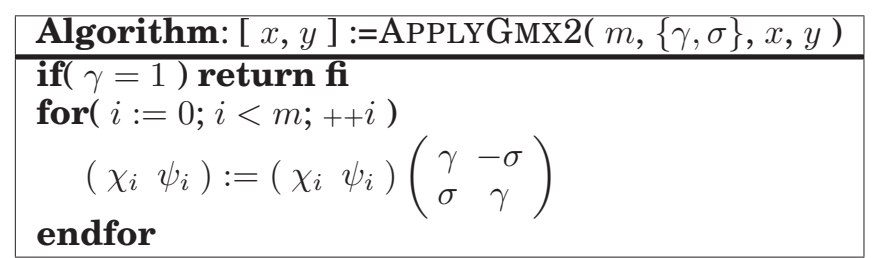

Fig. 5. An algorithm for applying a single Givens rotation, defined by a pair of Givens scalars $\{\gamma, \sigma\}$, to two length $m$ columns $x$ and $y$ from the right.

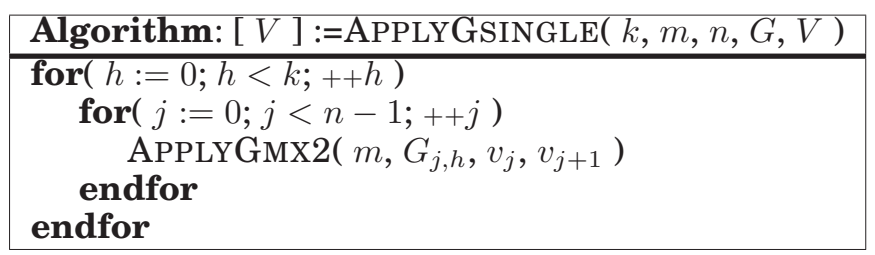

Fig. 6. An algorithm for applying the Givens rotations contained within an $(n-1) \times k$ matrix $G$ of Givens scalar pairs, in single-step fashion, to an $m \times n$ matrix $V$.

First, let us define a helper algorithm ApPLYGMX2, shown in Figure 5, which applies a single rotation $G=\left(\begin{array}{cc}\gamma & -\sigma \\ \sigma & \gamma\end{array}\right)$ from the right to a pair of vectors $x$ and $y$ of length $m$. The rotation is never explicitly formed. Rather, it is represented and passed in by the pair of Givens scalars $\{\gamma, \sigma\}$. Also, notice that APPLYGMX2 avoids any computation if the rotation is equal to identity.

Let us now formulate, for reference, an algorithm APPLYGSINGLE that, given an $(n-1) \times k$ matrix $G$ of Givens scalar pairs, applies the sets of Givens rotations in single-step fashion to an $m \times n$ matrix $V$. We show this algorithm in Figure 6 .

Figure 7 shows a wavefront algorithm that applies the Givens rotations stored in an $(n-1) \times k$ matrix $G$ of Givens scalars to an $m \times n$ matrix $V$. This algorithm implements the exact sequence of rotation application captured by the drawing in Figure 4. Each outer loop iterates through a series of waves, while the inner loops iterate through the rotations within each wave. Note that we simply call APPLYGSINGLE if there are not enough columns in $V$ to at least fully execute the startup and shutdown phases (ie: if $n<k)$.

\subsection{Blocking through $V$ to control cache footprint}

If applied to a matrix $V$ with $n$ rows, the wavefront algorithm requires approximately $k$ columns to simultaneously fit in cache. Clearly, if $k$ such columns do not fit, matrix $V$ can be partitioned into blocks of rows (using a blocksize $b$ ) and the $k$ sets of rotations can be applied to one such block at a time. Note that this blocking also naturally supports updating matrix $V$ in parallel by assigning blocks of rows to different processing cores.

\subsection{Why we avoid (for now) a hybrid QR/QL algorithm}

There exist certain kinds of graded matrices for which the $\mathrm{QR}$ algorithm does not perform well. In particular, if a matrix is graded so that $\left|\tau_{n-1, n-1}\right| \gg\left|\tau_{0,0}\right|$, then the shifting of the matrix (computing $\tau_{0,0}-\tau_{n-1, n-1}$ wipes out the accuracy in $\tau_{0,0}$ and causes excessive roundoff error. For this reason, an implementation may choose to

not new and has been used, for example, in the contexts of tridiagonalization via Householder-based band reduction [Bischof et al. 1994] and Givens-based band reduction within sparse SVD [Rajamanickam 2009]. 


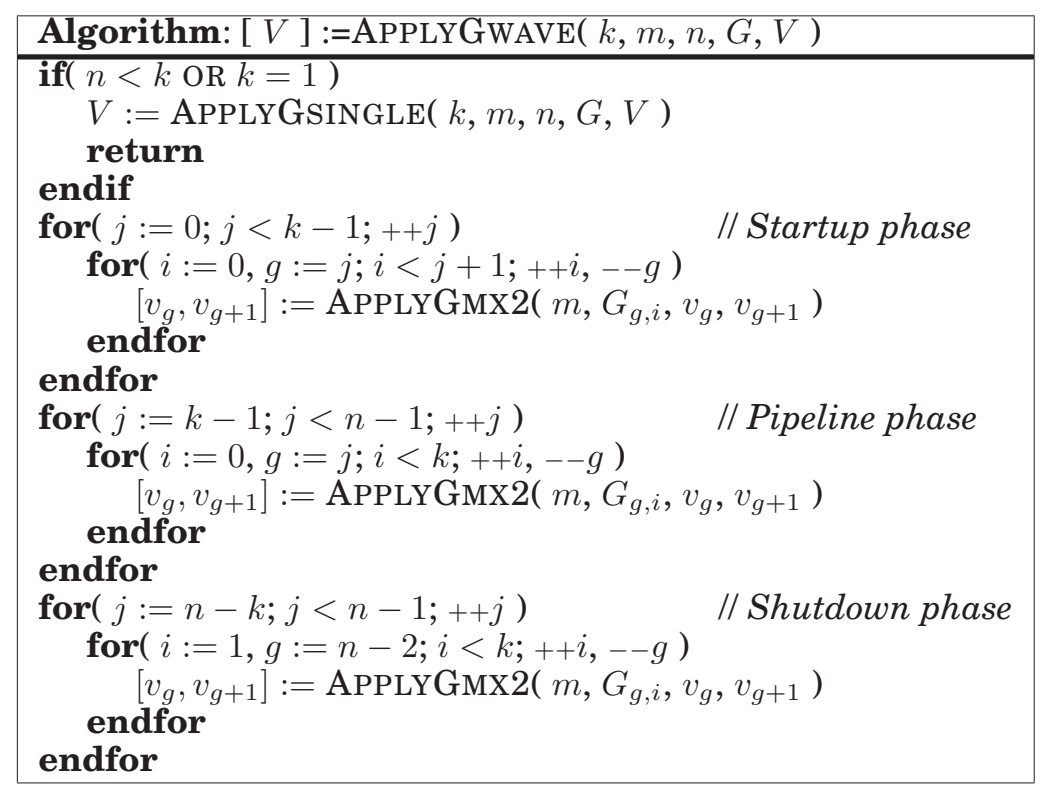

Fig. 7. An algorithm for applying the Givens rotations contained within an $(n-1) \times k$ matrix $G$ of Givens scalar pairs, in waves, to an $m \times n$ matrix $V$.

use a QL algorithm instead, in which $T-\kappa_{i} I \rightarrow Q L$ and $T^{\text {next }}:=L Q+\kappa_{i} I$. Indeed, depending on which of $\tau_{0,0}$ and $\tau_{n-1, n-1}$ is greater in magnitude, QR or QL may be used on an iteration-by-iteration basis.

Alternating between QR and QL steps disrupts our ability to accumulate and apply many rotations. Key to applying the wavefront algorithm is that all Francis steps move in the same direction through matrix $T$ : in the case of the $\mathrm{QR}$ algorithm, from topleft to bottom-right. Clearly, if we only used the QL algorithm, we could formulate a similarly optimized algorithm with waves moving in the opposite direction. It would also be possible to periodically switch between multiple QR steps and multiple QL steps. However, frequent mixing of directions would limit our ability to reuse data in cache. It is for this reason that we don't alternate between QR and QL steps. As a result, this is the one part of the traditional implementation that we do not yet directly incorporate in our restructured algorithm.

\section{A RESTRUCTURED TRIDIAGONAL QR ALGORITHM}

In the last section, we discussed how the application of multiple Francis sets of rotations can, in principle, yield a performance boost. The problem is that the QR algorithm, as implemented in LAPACK, is not structured to produce multiple sets of rotations. In this section, we discuss the necessary restructuring.

\subsection{The basic idea}

The fundamental problem is that applying multiple sets of rotations is most effective if there are a lot of rotations to be applied. Now, take a possible state of $T$ after some Francis steps have been performed:

$$
T=\left(\begin{array}{ccc}
T_{0} & 0 & 0 \\
0 & T_{1} & 0 \\
0 & 0 & T_{2}
\end{array}\right)
$$




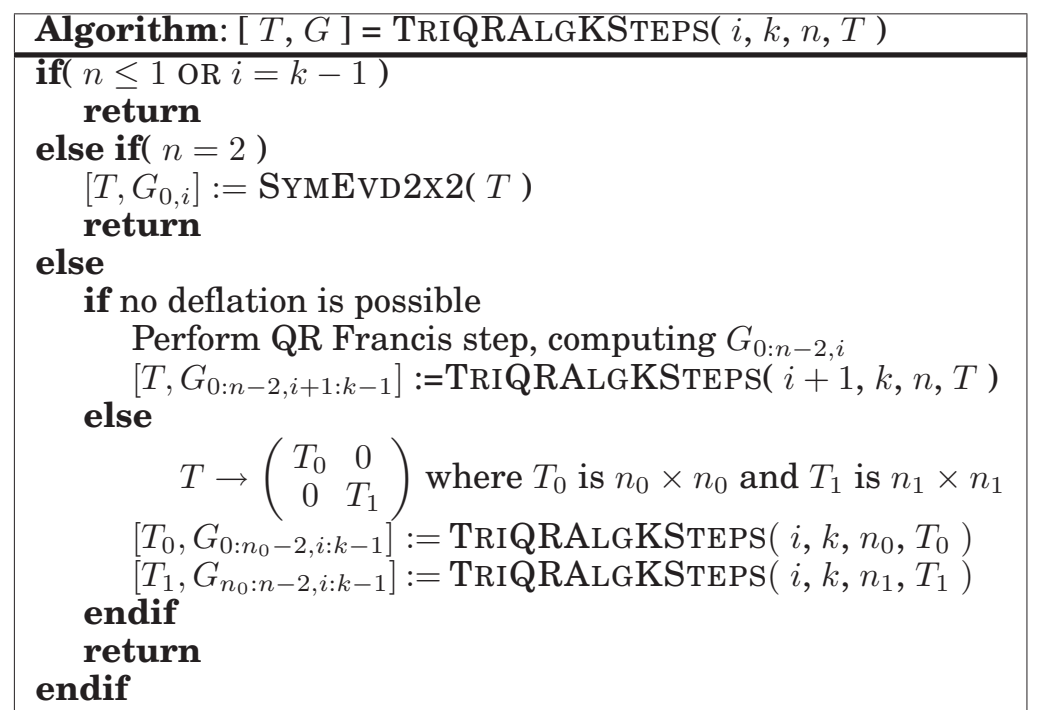

Fig. 8. A recursive algorithm for computing $k$ Francis steps, saving the rotations in $G$. It is assumed that $G$ was initialized with identity rotations upon entry.

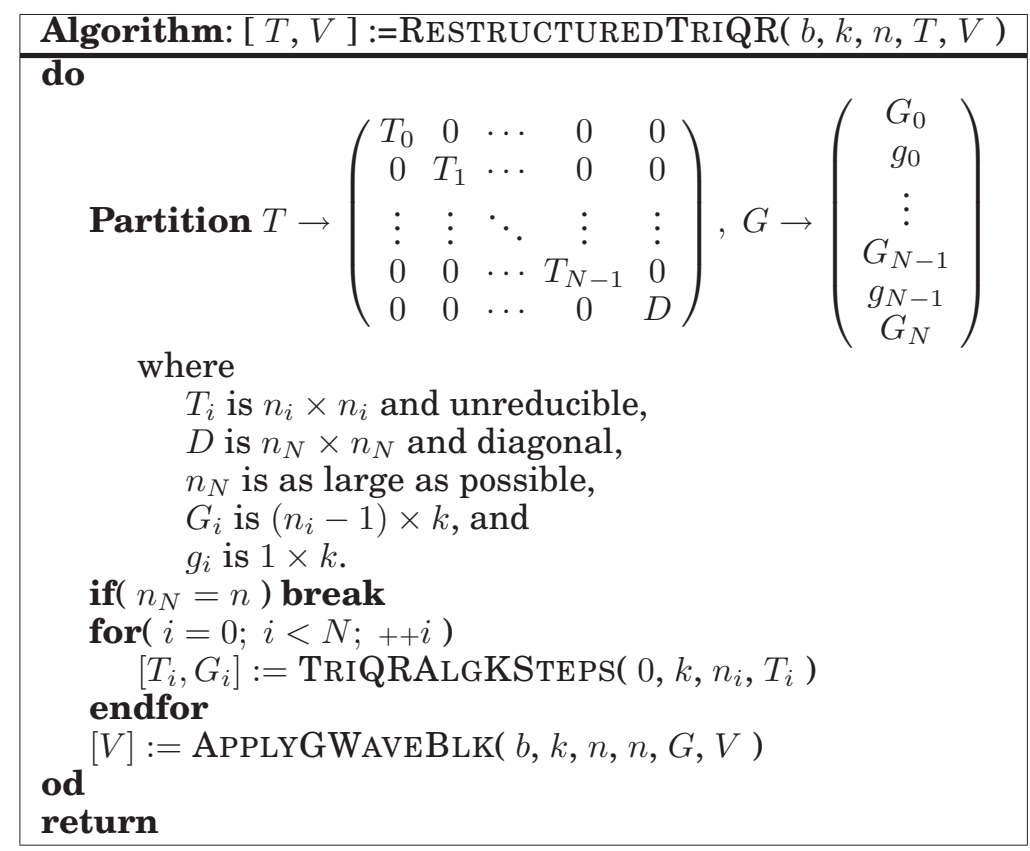

Fig. 9. Our restructured $Q R$ algorithm for the tridiagonal EVD.

What we capture here is the fact that an internal off-diagonal element may become negligible, causing a split. What a typical QR algorithm would do is continue separately with each of the diagonal blocks, but that would disrupt the generation of all rotations for all $k$ Francis steps with the original matrix $T$. We still want to take advantage of splitting. Essentially, if a split occurs and the number of Francis steps completed so far is $i<k$, then for each block on the diagonal we perform the remaining 
$k-i$ Francis steps, while continuing to accumulate rotations. After the $k$ rotation sets are accumulated for all subproblems along the diagonal of $T$, the rotations are applied to $V$. A recursive algorithm that achieves this is given in Figure 8. What is left is an outer loop around this routine, as given in Figure 9.

\subsection{Restructured $Q R$ algorithm details}

Now that we have given a brief, high-level description for the restructured tridiagonal $\mathrm{QR}$ algorithm, let us walk through the algorithm in further detail.

We start with the RESTRUCTUREDTRIQR algorithm in Figure 9. The first time through the outer loop, it is assumed that $T$ has no negligible (or zero) off-diagonal entries. Thus, $N=1, n_{0}=n, n_{N}=0$, and as a result $T$ is partitioned such that $T_{0}$ contains all of $T$ and $D$ is empty. Likewise, $G$ is partitioned into just the $(n-1) \times k$ subpartition $G_{0}$, which contains all of $G$. (Note that, once a split occurs, the reason we separate $G_{i}$ from $G_{i+1}$ by a single row vector $g_{i}$ is because this vector corresponds to the rotations that would have been applied to the $2 \times 2$ submatrix starting at element $\left(n_{i}-1, n_{i}-1\right)$ along the diagonal of $T$ if the split had not already occurred there.) Now, since $N=1$, the inner loop executes just once, calling TRIQRALGKSTEPS with $T_{0}=T$ and passing in $i=0$.

Within TRIQRALGKSTEPS, we return if $T$ is empty or a singleton. (We also return if $i=k-1$, which happens when we have accumulated the Givens scalars for $k$ Francis sets.) If $n=2$, we compute the EVD of $T$ via SYMEvD2X2, which returns the eigenvalues $\lambda_{1}$ and $\lambda_{2}$ along the diagonal of $T$ and a pair $\{\gamma, \sigma\}$ (stored to $G$ ) such that $T=\left(\begin{array}{cc}\gamma & -\sigma \\ \sigma & \gamma\end{array}\right)\left(\begin{array}{cc}\lambda_{1} & 0 \\ 0 & \lambda_{2}\end{array}\right)\left(\begin{array}{cc}\gamma & -\sigma \\ \sigma & \gamma\end{array}\right)^{T}$. Otherwise, if $n>2$, we search the off-diagonal of $T$ for negligible elements. If we find a negligible element, then we may split $T$ into topleft and bottom-right partitions $T_{0}$ and $T_{1}$, which are $n_{0} \times n_{0}$ and $n_{1} \times n_{1}$, respectively, where $n_{0}+n_{1}=n$, and then call TRIQRALGKSTEPS recursively on these subpartitions, saving the resulting Givens scalar pairs to the corresponding rows of columns $i$ through $k-1$. If no negligible off-diagonal elements are found, then we perform a Francis step on $T$, saving the Givens scalar pairs to the $i$ th column of matrix $G$. We then proceed by recursively calling TRIQRALGKSTEPS with an incremented value of $i$.

At some point, we will fill matrix $G$ with Givens scalar pairs, at which time control returns to RESTRUCTUREDTRIQR and APPLYGWAVEBLK applies the rotations encoded in $G$ to matrix $V$.

After the application, the outer loop repeats and, upon re-entering the inner loop, another "sweep" of $T$ begins where up to $k$ Francis steps are performed on each submatrix $T_{i}$ before once again applying the rotations to $V$. This process repeats until the entire off-diagonal of $T$ becomes negligible, at which time $n_{N}=n$, and control breaks out of the outer loop, signaling that the QR algorithm's execution is complete.

\subsection{Impact of identity rotations}

Notice that we initialize $G$ to contain identity rotations $(\gamma=1$ and $\sigma=0)$ prior to each entry into the inner loop of RESTRUCTUREDTRIQR. This allows Francis steps executed by TRIQRALGKSTEPS to simply overwrite elements of $G$ with scalar pairs representing Givens rotations as they are computed, without having to worry about which elements are not needed due to deflation (and splitting). Then, APPLYGWAVE simply detects these identity rotations and skips them so as to save computation.

Depending on how much splitting occurs, this method of storing rotations could have an impact on cache performance. We would expect that the wavefront algorithm for applying Givens rotations would execute most favorably when splits occur only (or 


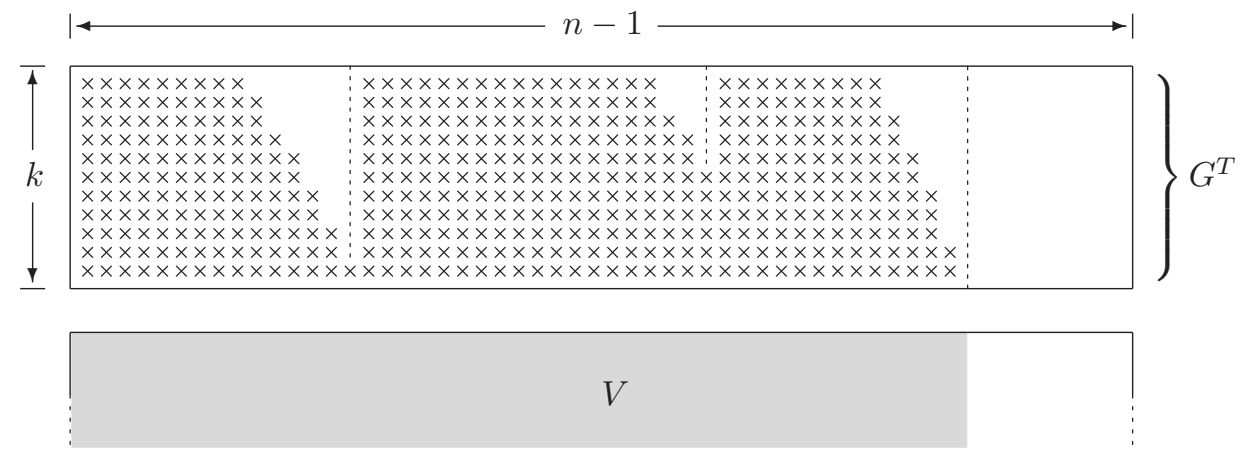

Fig. 10. An illustration of the possible contents of $(n-1) \times k$ matrix $G$ at the end of a sweep, just before its rotations are applied to matrix $V$. ( $G$ is shown transposed to better visualize the portion of $V$ (highlighted) that is affected by the application.) Givens scalar pairs, which encode Givens rotations, are marked by " $\times$ " symbols while unmarked locations represent identity rotations (which are skipped). This picture shows what $G$ might look like after executing a sweep that encountered two instances of splitting: one in the first Francis step, which occurred while iterating over the entire non-deflated portion of $T$, and one in the sixth Francis step, which occurred within the lower-right recursive subproblem that began as a result of the first split.

mostly) at or near the bottom-right corner of $T$. This is because each column of $V$ would be accessed and re-used a maximum number of times.

However, Figure 10 shows how splitting early on in a set of Francis steps can create "pockets" of identity rotations. When these identity rotations are skipped, the columns associated with these rotations are not touched. This results in a somewhat greater proportion of cache misses since the reduction in overall column-accesses comes entirely from a reduction in column-accesses that would have been cache hits. The worst case arises when a split, and subsequent deflation, occurs (1) somewhere in the middle of $T$, corresponding to the columns that would have been updated as part of full waves, and/or (2) very early on in the Francis set, as it gives the pocket of identity rotations the most time to grow due to subsequent tail deflation in the upper-left subproblem.

\section{OPTIMIZING THE APPLICATION OF GIVENS ROTATIONS}

In Section 4, we developed a wavefront algorithm for applying Givens rotations that is, in theory, capable of accessing data in a very cache-efficient manner. We also introduced a supplementary blocked algorithm to partition the eigenvector matrix $V$ into smaller panels to further control the amount of data from $V$ the algorithm tries to keep in cache without compromising the expected hit rate for column-accesses. This effectively induces a blocked algorithm for the operation, which constitutes a substantial algorithm-level improvement over a naive approach that applies one Francis set of rotations at a time to the entire matrix $V$. Even with these advances, further optimizations are possible.

\subsection{Instruction-level optimizations}

Instead of implementing the wavefront algorithm implementation in traditional $\mathrm{C}$ and relying only upon the compiler to perform instruction-level optimizations, we instead utilize SSE vector intrinsics to facilitate the use of SIMD hardware instructions. These low-level, vector-based APIs are supported by recent versions of both the Intel and GNU C compilers, and allow the software developer to precisely specify when to load and store data. In our case, the vector intrinsic code was applied only to the implementation of APPLYGMX2 (and derivatives mentioned in the next section). The curious 


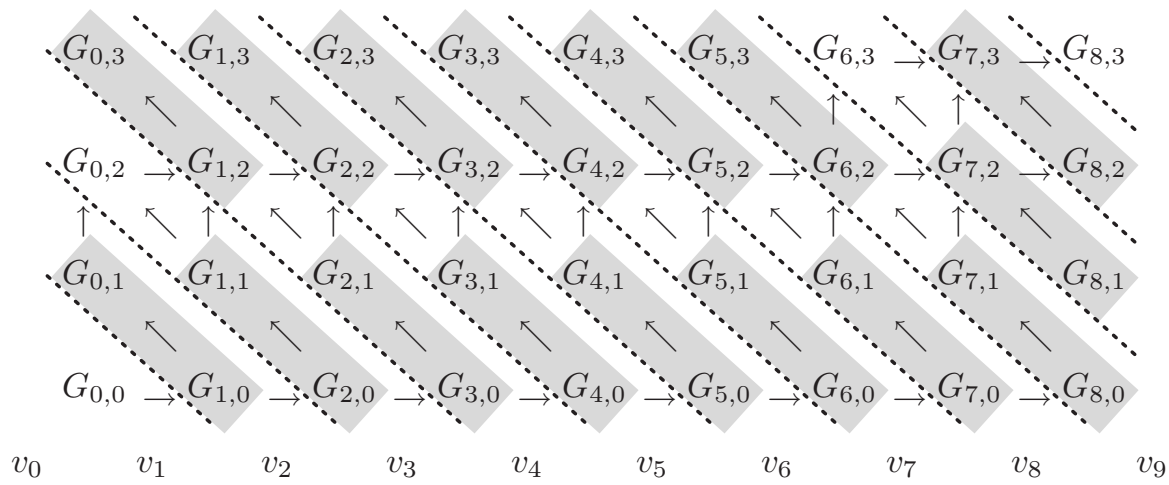

Fig. 11. By fusing pairs of successive rotations within the same wave, the number of memory operations performed on each element of $V$, per rotation applied, may be reduced from approximately four to three. Here, fused rotations are highlighted, with arrows redrawn to show dependencies within a fused pair as well as dependencies between pairs. Rotation pairs may be applied in waves, similar to the approach illustrated by Figure 4 . Note that the updated dependencies satisfy the original dependency graph.

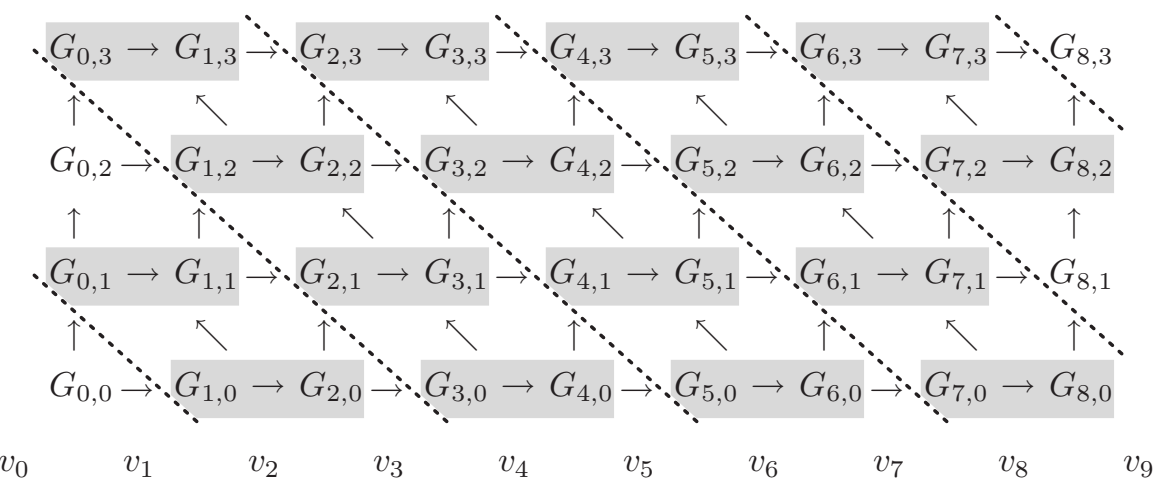

Fig. 12. Instead of fusing successive rotations within the same wave, as shown in Figure 11, we may also fuse pairs of successive rotations from the same Francis step. This approach similarly reduces the number of memory operations performed on each element of $V$ per rotation from four to three.

reader can find a concrete code example in a related technical report [Van Zee et al. $2011]$.

Next, we will show how precisely controlling the loading and storing of data to and from main memory opens the door to further algorithmic-level optimizations within the application of Givens rotations.

\subsection{Fusing rotations}

In [Van Zee et al. 2012] the authors showed how certain level-2 BLAS operations could be "fused" to reduce the total number of memory accesses, which tend to be rather slow and expensive relative to floating-point computation. They further showed that fusing two operations at the register-level- that is, re-using the data while it is still loaded in the register set-is superior to a cache-level approach whereby we simply assume that recently touched data in the cache will be readily available. The bottom line: all else being equal, reducing the number of memory operations, regardless of whether the data is already in cache, will typically allow shorter execution times and thus higher performance. 


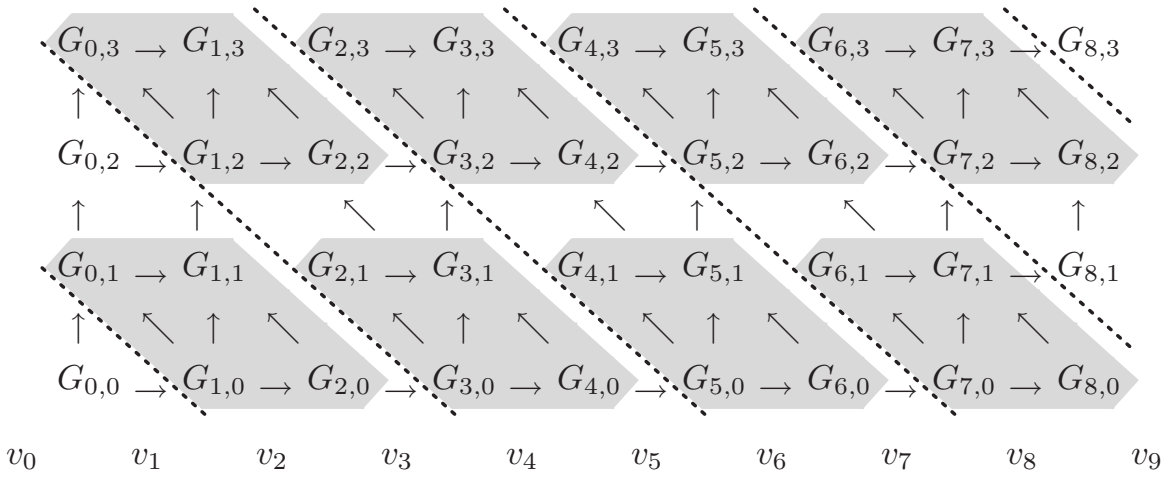

Fig. 13. Combining the approaches from Figures 11 and 12 , we can fuse in both the $k$ and $n$ dimensions. This creates parallelogram-shaped groups of fusings. This two-dimensional fusing reduces the average number of column-access memory operations per rotation from four, in an algorithm that uses no fusing, to just two.

Consider Figure 11. Here, we have made the observation that successive Givens rotations within a single wave may be fused to reduce the number of times the rotations must access their corresponding columns of $V$. (Dependency arrows have been redrawn to indicate dependencies within pairs of rotations as well as dependencies between pairs.) Let us consider one such fusable pair, $G_{1,0}$ and $G_{0,1}$, in detail. When no fusing is employed, $G_{1,0}$ will load and store each element of $v_{1}$ and $v_{2}$, for a total of four column-accesses. Immediately following, $G_{0,1}$ will load and store $v_{0}$ and $v_{1}$, for another four column accesses. However, any given element of $v_{1}$ does not change in between the time that $G_{1,0}$ stores it and $G_{0,1}$ loads it. Thus, we could fuse the application of rotations $G_{1,0}$ and $G_{0,1}$. This would result in the $i$ th element of $v_{1}$ and the $i$ th element of $v_{2}$ first being loaded (by $G_{1,0}$ ). After computing the updated values, only element $i$ of $v_{2}$ is stored while the $i$ th element of $v_{1}$ is retained in a register. At this point, $G_{0,1}$ need only load the corresponding $i$ th element from $v_{0}$ in order to execute. After its computation, $G_{0,1}$ stores the $i$ th elements of both $v_{0}$ and $v_{1}$. This reduces the total number of column-accesses needed to apply two rotations from eight to six. Put another way, this reduces the number of column-accesses needed per rotation from four to three-a 25 percent reduction in memory operations. Likewise, this increases the ratio of total flops to total memops from 1.5, as established in Section 4.1, to 2.

We can express the number of memory accesses in terms of the level of fusing, as follows. If we fuse $p$ rotations in a single wave where $p \ll k$, then the approximate number of total column-accesses incurred is given by $2 k n\left(1+\frac{1}{p}\right)$, and thus with $k(n-1)$ total rotations to apply, the number of column-accesses needed per rotation is approximated by $2\left(1+\frac{1}{p}\right)$. Notice that aggressively increasing $p$ results in diminishing improvement in the number of memory accesses.

If we turn our attention back to Figure 4, we can find another method of fusing rotation pairs. This method is illustrated in Figure 12. Instead of fusing successive rotations within the same wave, we can instead fuse successive rotations from the same Francis step. This approach, like the previous one, is similarly generalizable to fuse $p$ rotations, and similarly reduces the number of column memory operations incurred per rotation to $2\left(1+\frac{1}{p}\right)$.

Now, let us revisit both Figures 11 and 12. Notice that the dependency arrows allow us to fuse rotations along both the $k$ dimension (across Francis sets) and the $n$ 
dimension (within a Francis set) of matrix $G$, as illustrated by Figure 13, to create parallelogram-shaped groups of fused rotations. By inspection, we find that the average number of column-accesses per rotation, $n_{C A P R}$, incurred by this more generalized method of fusing can be expressed as

$$
n_{C A P R} \approx \frac{2\left(p_{k}+p_{n}\right)}{p_{k} p_{n}}
$$

where $p_{k}$ and $p_{n}$ are the number of rotations fused in the $k$ and $n$ dimensions, respectively. Notice that for $p_{k}=p_{n}=2$, the situation captured by Figure 13, this two-dimensional fusing reduces the average number of memory operations required per rotation to just two column-accesses. This places the ratio of total flops to total memops at 3. Also notice that the optimal fusing shape is that of a "square" parallelogram (ie: $p_{k}=p_{n}$ ) because it corresponds to the classic problem of maximizing the area of a rectangle (the number of rotations) relative to its perimeter (the number of column-accesses).

In the limit, when $p_{k}=k$ and $p_{n}=n$, the ratio of total flops to memops rises to our theoretical maximum, $3 k$. However, values of $p_{k}$ and $p_{n}$ higher than 2 are harder to implement because it implies that either (1) an increasing number of Givens scalars are being kept in registers, or (2) an increasing number of Givens scalars' load times are being overlapped with computation (a technique sometimes called "pre-fetching"). Since the processor's register set is typically quite small, either of these situations may be sustained only to a limited extent.

\subsection{Pitfalls to fusing}

In our experience, we have encountered one major caveat to fusing: if any of the rotations within a fused group is the identity rotation, then extra conditional logic must be introduced into the algorithm so as to not unnecessarily apply that rotation to $V$. This extra logic may add some overhead to the algorithm. In addition, the larger the fused group of rotations, the greater the chance that any given fused group will contain an identity rotation, which reduces opportunities for cache reuse during the execution of that group.

\subsection{An alternate method of forming $Q$ for complex matrices}

Until now, we have only discussed Hermitian EVD whereby Givens rotations computed during the $\mathrm{QR}$ iteration are applied directly to $V=Q_{A}$, where $Q_{A} \in \mathbb{C}^{n \times n}$. However, there is another option that holds potential as long as the input matrix $A$ remains complex.

Consider an algorithm for EVD in which we initialize $V_{R}=I$, where $V_{R} \in \mathbb{R}^{n \times n}$. Rotations may then be accumulated into $V_{R}$ as before, which results in $V_{R}=Q_{T}$. Subsequently, $Q$ may be formed by computing $Q:=Q_{A} V_{R}$, which may be done with a general matrix-matrix multiplication where the left input matrix is complex and the right input matrix is real. While this kind of dgemm is not implemented in the BLAS, it is equivalent to multiplying $V_{R}$ by the real and imaginary components of $Q_{A}$ separately. If all matrices are stored in column-major order, this can be done by using dgemm and specifying the $m$ and leading dimensions of $Q_{A}$ as twice their actual values, since the floating-point representation of complex numbers consists of two real-valued components. This requires that $Q_{A}$ be copied to a temporary matrix $\hat{Q}_{A}$ so that dgemm can overwrite $Q_{A}:=\hat{Q}_{A} V_{R}$. This approach has advantages and at least one major drawback.

The first advantage of this approach is that part of the computation is now shifted to dgemm, which is capable of near-peak performance on many modern architectures [Goto 
and van de Geijn 2008] (whereas applying Givens rotations is inherently limited to $75 \%$ of peak on some architectures, as discussed later in Section 9.2).

The second advantage is that this method incurs fewer floating-point operations when the number of Francis steps performed per deflation, $f$, is above a certain threshold. As discussed earlier in this paper, applying rotations directly to $V=Q_{A}$ leads to approximately $2 \times 3 f n^{3}$ flops. However, accumulating the rotations to a real matrix $V_{R}$ results in only $3 \mathrm{fn}^{3}$ flops. The subsequent invocation of dgemm incurs an additional $2(2 n) n^{2}=4 n^{3}$ flops. Thus, this approach incurs fewer flops when $3 f n^{3}+4 n^{3}<2 \times 3 f n^{3}$, or, when $f>\frac{4}{3}$. However, additional flops may be saved during the accumulation of $V_{R}$. Notice that after applying the first Francis set of rotations to $V_{R}=I$, the matrix is filled in down to the first subdiagonal with the remaining entries containing zeros. Applying the second Francis set results in one additional subdiagonal of fill-in. By exploiting this gradual fill-in, we can save approximately $n^{3}$ flops, reducing the total number of Givens rotation flops from $3 f n^{3}$ to approximately $(3 f-1) n^{3}$. With these flop savings, the method now incurs fewer flops when $f>1$, which is almost always the case.

The most obvious disadvantage of this approach is that it requires $\mathcal{O}\left(n^{2}\right)$ workspace. Specifically, one would need to allocate a real $n \times n$ matrix $V_{R}$ to store the accumulated rotations. In addition, it would require a second complex $n \times n$ matrix to hold $\hat{Q}_{A}$. (Actually, one could get by with allocating a smaller $b \times n$ matrix for $\hat{Q}_{A}$, where $b \ll n$ but still large enough to allow dgemm to operate at high performance, and use a blocked algorithm to repeatedly perform several smaller panel-matrix multiplications.)

The idea of using matrix-matrix multiplication to accelerate rotation-based algorithms has been studied before [Lang 1998; Quintana-Ortí and Vidal 1992]. Most recently, in [Kågström et al. 2008], it has been shown that it is possible to accumulate blocks of rotations and then use dgemm and dtrmm to multiply these blocks into $V=Q_{A}$. This method uses only $\mathcal{O}(n)$ workspace. However, this method incurs roughly $50 \%$ more flops than our plain restructured QR algorithm due to overlap between the accumulated blocks. This method would become advantageous when the ratio of observed performance between APPLYGWAVEBLK and dgemm (and dtrmm) is less than $\frac{2}{3}$. Otherwise, we would expect this method to exhibit longer run times than that of applying rotations directly to $V=Q_{A}$ via the blocked wavefront algorithm. (In Section 9.2, we report the performance of an implementation of APPLYGWAVEBLK that does indeed exceed this two-thirds threshold relative to dgemm on the test machine.)

\section{NUMERICAL ACCURACY}

Ordinarily, this kind of paper would include extensive experiments to measure the accuracy of the new approach and compare it to that of other algorithms. However, because the computations performed by our restructured $\mathrm{QR}$ algorithm, and the order in which they are performed, are virtually identical to those of the original implementation in LAPACK (modulo the case of certain highly graded matrices), it follows that the algorithm's numerical properties are preserved, and thus the residuals $\left\|A v_{j}-\lambda_{j} v_{j}\right\|$ are small. Furthermore, the accuracy and convergence properties of the tridiagonal QR algorithm relative to other algorithms are well-studied subjects [Wilkinson 1968; Stewart 2001; Demmel et al. 2008].

It is worth pointing out that even in the case of graded matrices, there are certain cases where the $\mathrm{QR}$ algorithm performs well. Specifically, if the matrix grading is downward along the diagonals, the $\mathrm{QR}$ algorithm typically yields eigenvalues and eigenvectors to high relative accuracy [Stewart 2001]. 


\section{EXTENDING TO SINGULAR VALUE DECOMPOSITION}

Virtually all of the insights presented in this paper thus far may be easily applied to the SVD of a dense matrix.

Given a general matrix $A \in \mathbb{C}^{m \times n}$, its singular value decomposition is given by $A=U \Sigma V^{H}$, where $U \in \mathbb{C}^{m \times m}, V \in \mathbb{C}^{n \times n}$, both $U$ and $V$ are unitary, and $\Sigma \in \mathbb{R}^{m \times n}$ is positive and diagonal. The singular values of $A$ can then be found on the diagonal of $\Sigma$ while the corresponding left and right singular vectors reside in $U$ and $V$, respectively.

Computing the SVD is similar to the three stages of dense Hermitian EVD [Stewart 2001]: reduce matrix $A$ to real bidiagonal form $B$ via unitary similarity transformations: $A=U_{A} B V_{A}^{H}$; compute the SVD of $B: B=U_{B} \Sigma V_{B}^{T}$; and back-transform the left and right singular vectors of $B: U=U_{A} U_{B}$ and $V=V_{A} V_{B}$ so that $A=U \Sigma V^{H}$.

If $m$ is larger than $n$ to a significant degree, it is often more efficient to first perform a QR factorization $A \rightarrow Q R$ and then reduce the upper triangular factor $R$ to real bidiagonal form [Stewart 2001], as $R=U_{R} B V_{R}^{H}$. The process then unfolds as described above, except that after the bidiagonal SVD (and back-transformation, if applicable), the matrix $U_{R} U_{B}$ must eventually be combined with the unitary matrix $Q$ to form the left singular vectors of $A$.

Let us briefly discuss each stage.

Reduction to real bidiagonal form. One can reduce a matrix to bidiagonal form in a manner very similar to that of tridiagonal form, except that here separate Householder transformations are computed for and applied to the left and right sides of $A$. In the (more common) case of $m \geq n$, where the matrix is reduced to upper bidiagonal form, these transformations annihilate the strictly lower triangle and all elements above the first superdiagonal. Families of algorithms for reducing a matrix to bidiagonal form are given in [Van Zee et al. 2012].

Computing the SVD of a real bidiagonal matrix. Computing the SVD of a real bidiagonal matrix can be performed via Francis steps similar to those discussed in Section 3.2, except that: (1) the shift is typically computed from the smallest singular value of the trailing $2 \times 2$ submatrix along the diagonal [Stewart 2001]; (2) the bulge in the Francis step is chased by computing and applying separate Givens rotations from the left and the right; and (3) the left and right Givens rotations are similarly applied to left and right matrices which, upon completion, contain the left and right singular vectors of the bidiagonal matrix $B$. Also, the test to determine whether a superdiagonal element is negligible is somewhat different than the test used in the tridiagonal QR algorithm. Details concerning the negligibility test, along with other topics related to computing singular values, may be found in [Demmel and Kahan 1990]. Others have successfully developed Divide and Conquer [Gu and Eisenstat 1995a] and MRRR [Willems et al. 2006] algorithms for computing the singular value decomposition of a bidiagonal matrix. The most recent release of LAPACK (version 3.3.1 as of this writing) includes an implementation of dense matrix SVD based on the D\&C algorithm; however, an MRRR implementation has not yet been published in the library. Many algorithmic variants of the SVD exist, including several based on the QR algorithm [Cline and Dhillon 2006], but for practical purposes we limit our consideration to those algorithms implemented in LAPACK.

Back-transformation of $U$ and $V$. The back-transformation of $U$ and $V$ take place in a manner similar to that of $Q$ in the EVD, except that when $m>n$ only the first $n$ columns of $U_{A}$ are updated by $U_{B}$, and when $m<n$ only the first $m$ columns of $V_{A}$ are updated by $V_{B}$.

Figures 14 and 15 show floating-point operation counts and workspace requirements, respectively, for the dense SVD problem. When counting workspace for SVD, we 


\begin{tabular}{|l|c|c|c|}
\hline \multirow{2}{*}{$\begin{array}{l}\text { SVD } \\
\text { Algorithm }\end{array}$} & \multicolumn{3}{|c|}{ Effective higher-order floating-point operation cost } \\
\cline { 2 - 4 } & $A \rightarrow U_{A} B V_{A}^{H}$ & $B \rightarrow U_{B} \Sigma V_{B}^{T}$ & Form/Apply $U, V$ \\
\hline orig. QR & $\begin{array}{l}4 \times\left(K_{2}+1\right) \\
\left(2 m n^{2}-\frac{2}{3} n^{3}\right)\end{array}$ & $2 \times K_{1} 3 f n^{2}(m+n)$ & $4 \times 4 n\left(m^{2}-m n+\frac{2}{3} n^{2}\right)$ \\
\hline rest. QR & $\begin{array}{l}4 \times\left(K_{2}+1\right) \\
\left(2 m n^{2}-\frac{2}{3} n^{3}\right)\end{array}$ & $2 \times 3 f n^{2}(m+n)$ & $4 \times 4 n\left(m^{2}-m n+\frac{2}{3} n^{2}\right)$ \\
\hline D\&C & $\begin{array}{l}4 \times\left(K_{2}+1\right) \\
\left(2 m n^{2}-\frac{2}{3} n^{3}\right)\end{array}$ & $\mathcal{O}\left(m n+n^{2}\right)$ & $4 \times 4 n\left(m^{2}-\frac{1}{2} m n+\frac{1}{2} n^{2}\right)$ \\
\hline
\end{tabular}

Fig. 14. A summary of approximate floating-point operation cost for suboperations of various algorithms for computing a general singular value decomposition. Integer scalars at the front of the expressions (e.g. " $4 \times \ldots$...) indicate the floating-point multiple due to performing arithmetic with complex values. In the cost estimates for the QR algorithms, $f$ represents the typical number of Francis steps needed for an eigenvalue to converge. For simplicity, it is assumed that $m \geq n$, but also that it is not the case that $m \gg n$ and thus an initial QR factorization of $A$ is not employed.

\begin{tabular}{|l|l|l|c|}
\hline \multirow{2}{*}{ SVD Algorithm } & \multicolumn{3}{|c|}{ Workspace required for optimal performance } \\
\cline { 2 - 4 } & \multicolumn{1}{|c|}{$A \rightarrow U_{A} B V_{A}^{H}$} & $B \rightarrow U_{B} \Sigma V_{B}^{T}$ & Form/Apply $U, V$ \\
\hline original QR & $\begin{array}{l}\mathbb{C}: 2 n+b(m+n) \\
\mathbb{R}: 2 n-1\end{array}$ & $\mathbb{R}: 4(n-1)$ & $\mathbb{C}: b(m-1)$ \\
\hline restructured QR & $\begin{array}{l}\mathbb{C}: 2 n+b(m+3 n) \\
\mathbb{R}: 2 n-1\end{array}$ & $\mathbb{R}: 4 b(n-1)$ & $\mathbb{C}: b(m-1)$ \\
\hline D\&C & $\mathbb{C}: 2 n+b(m+n)$ & $\begin{array}{l}\mathbb{R}: 3 n^{2}+4 n \\
\mathbb{R}:: 8 n\end{array}$ & $\mathbb{C}: b m$ \\
\hline
\end{tabular}

Fig. 15. A summary of workspace required to attain optimal performance for each suboperation of various algorithms employed in computing a general singular value decomposition. For simplicity, it is assumed that $m \geq n$, but also that it is not the case that $m \gg n$ and thus an initial QR factorization of $A$ is not employed.

do not count either of the $m \times m$ or $n \times n$ output singular vector matrices as workspace. Here, we stray from our convention of only exempting the input matrices since it is not possible to output all singular vectors by overwriting the input matrix.

As with reduction to tridiagonal form, our reduction to bidiagonal form retains the triangular factors of the block Householder transformations, except here the operation requires an additional $2 b n$ complex storage elements since we must retain the triangular factors for both $U_{A}$ and $V_{A}$.

Note that the back-transformation of D\&C has an $\mathcal{O}\left(n^{3}\right)$ cost difference relative to the $\mathrm{QR}$ algorithm that is very similar to the cost difference found in the D\&C and MRRR algorithms for computing the Hermitian EVD. Actually, when $m=n$, the addtional cost is $\frac{4}{3} n^{3}$, which is twice as large as that of the EVD because the SVD must back-transform both $U$ and $V$. The original and restructured $\mathrm{QR}$ algorithms can achieve high performance with approximately $\mathcal{O}(b(m+n))$ workspace while the D\&Cbased method requires an additional $3 n^{2}$ workspace elements.

\section{PERFORMANCE}

We have implemented the restructured QR algorithm, along with several algorithms for applying multiple sets of Givens rotations, including several optimized variants of the wavefront algorithm presented in Section 4. In this section, we will discuss observed performance of these implementations. 


\subsection{Platform and implementation details}

All experiments reported in this paper were performed on a single core of a Dell PowerEdge R900 server consisting of four Intel "Dunnington" six-core processors, where each core has a 16 megabyte L2 cache. Each core provides a peak performance of 10.64 GFLOPS. Performance experiments were gathered under the GNU/Linux 2.6.18 operating system. All experiments were performed in double-precision floating-point arithmetic on complex Hermitian (for EVD) and complex general (for SVD) input matrices. Generally speaking, we test three types of implementations:

— netlib LAPACK. Our core set of comparisons are against Hermitian EVD and SVD implementations found in the netlib distribution of the LAPACK library, version 3.3.1 [Anderson et al. 1999; LAPACK 2011]. When building executables for these netlib codes, we link to OpenBLAS 0.1-alpha-2.4 [OpenBLAS 2011]. The OpenBLAS project provides highly-optimized BLAS based on the GotoBLAS2 library, which was released under an open source license subsequent to version 1.13.

- MKL. We also compare against the Math Kernel Library (MKL), version 10.2.2, which is a highly-optimized commercial product offered by Intel that provides a wide range of scientific computing tools, including an implementation of LAPACK as well as a built-in BLAS library.

- libflame. The restructured QR algorithm discussed in this paper was implemented in the $\mathrm{C}$ programming language and integrated into the libflame library for dense matrix computations, which is available to the scientific computing community under an open source license [Van Zee 2011; libflame 2011]. When linking these implementations, we use the same OpenBLAS library used to link netlib codes.

We compile the netlib and libflame codes with the GNU Fortran and C compilers, respectively, included in the GNU Compiler Collection version 4.1.2.

\subsection{Applying Givens rotations}

Before presenting dense EVD and SVD results, we will first review performance results for various implementations for applying $k$ sets of Givens rotations to an $n \times n$ matrix. For these tests, random (non-identity) rotations were applied to a random matrix $V$. The results for these implementations are shown in Figure 16.

Let us begin our discussion with the results for "ApplyGsingle (no SSE)," which most closely represent the performance of the LAPACK routine zlasr, which applies a single set of Givens rotations to a double-precision complex matrix. Asymptotically, the performance for this implementation is very low-on par with un-optimized level-1 BLAS routines. As with many so-called "unblocked" algorithms, this one performs somewhat better for smaller problem sizes, when the entire matrix $V$ can fit in the L2 cache. Looking at the results for "ApplyGsingle (with SSE)," we see the benefit to using SSE instructions (implemented via C compiler intrinsics). However, performance beyond small problems is still quite low due to the algorithm's relative cache-inefficiency, as discussed in Section 4. By switching to a wavefront algorithm with blocking (while still using SSE vector intrinsics), as reflected by "ApplyGwaveBlk (no fusing)," performance is significantly improved and quite consistent as problem sizes grow larger. We further optimize the blocked wavefront algorithm by employing three kinds of fusing (labeled as $p_{k} \times p_{n}$ ), corresponding to the illustrations in Figures 11, 12, and 13. Remarkably, fusing with $p_{k}=p_{n}=2$ yields performance that is over $60 \%$ higher than a similar blocked wavefront implementation without fusing. We include dense dgemm performance (via OpenBLAS) for reference, where all three matrices are square and 


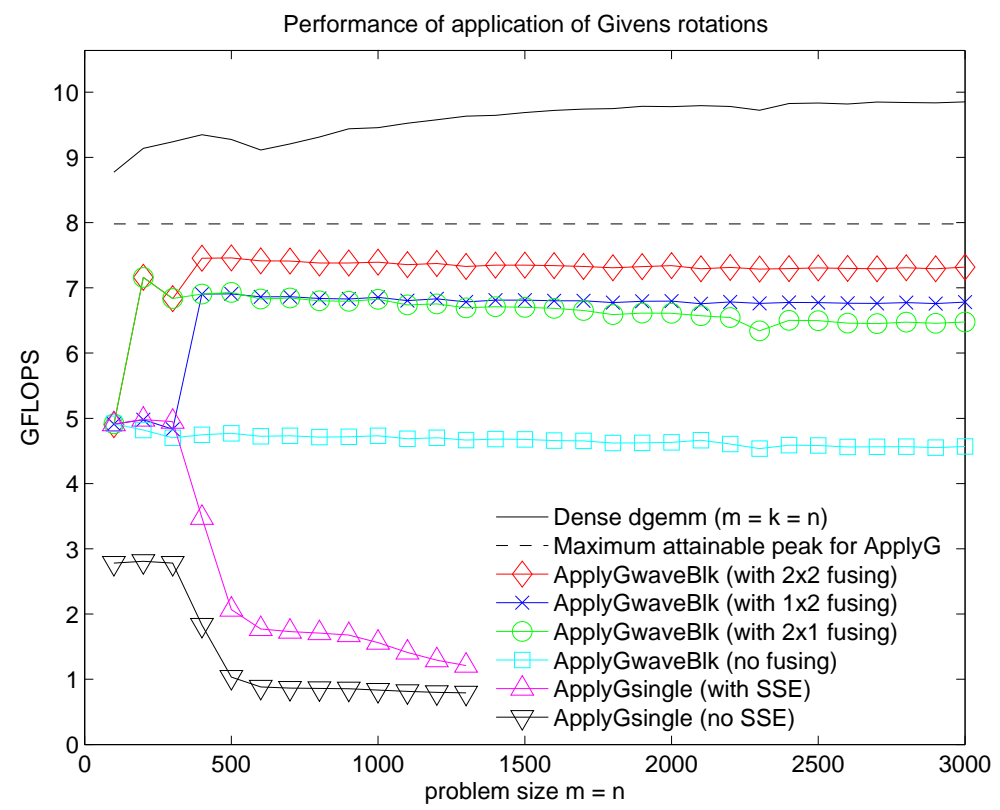

Fig. 16. Performance of various implementations for applying $k=192$ sets of Givens rotations. Those implementations based on blocked algorithms use an algorithmic blocksize $b=256$. The maximum attainable peak for applying Givens rotations, which is 25\% lower than that of dgemm because of a 1:2 ratio of floatingpoint additions to multiplications, is also shown. Performance results for dgemm (via OpenBLAS), where all matrix dimensions are equal, are included for reference.

equal to the problem size. We also mark the maximum attainable peak performance for applying Givens rotations. ${ }^{9}$

\subsection{Hermitian eigenvalue decomposition}

Figure 17 shows relative performance for eight implementations for Hermitian EVD, with linearly distributed eigenvalues. (We report results for five other distributions of eigenvalues in Appendix A, along with descriptions of how the distributions were created. There, we also report results for computation on real-domain matrices.) The implementations tested are described as follows:

- MKL EVD via QR refers to MKL zheev.

- MKL EVD via D\&C refers to MKL zheevd.

- MKL EVD via MRRR refers to MKL zheevr.

- netlib EVD via QR refers to netlib zheev linked to OpenBLAS.

— netlib EVD via D\&C refers to netlib zheevd ${ }^{10}$ linked to OpenBLAS.

— netlib EVD via MRRR refers to netlib zheevr linked to OpenBLAS.

${ }^{9}$ Matrix-matrix multiplication typically incurs equal numbers of floating-point additions and multiplica-
tions, which is well-suited for modern architectures which can execute one add and one multiply simultane-
ously. But if an operation does not have a one-to-one ratio of additions to multiplications, some instruction
cycles are wasted, resulting in a lower peak performance. The application of Givens rotations is one such
operation, generating one addition for every two multiplications. Furthermore, we confirmed that our test
system does indeed suffer from this limitation in floating-point execution. Thus, our maximum attainable
peak is 7.98 GFLOPS, which is $25 \%$ lower than the single core peak of 10.64 GFLOPS.
${ }^{10}$ During our tests, we discovered that the netlib implementation of zheevd, when queried for the optimal
amount of workspace, returns a value that is approximately $b(n-1)$ less than is needed for the back-
transformation to run as a blocked algorithm, which unnecessarily limits performance for most problem 


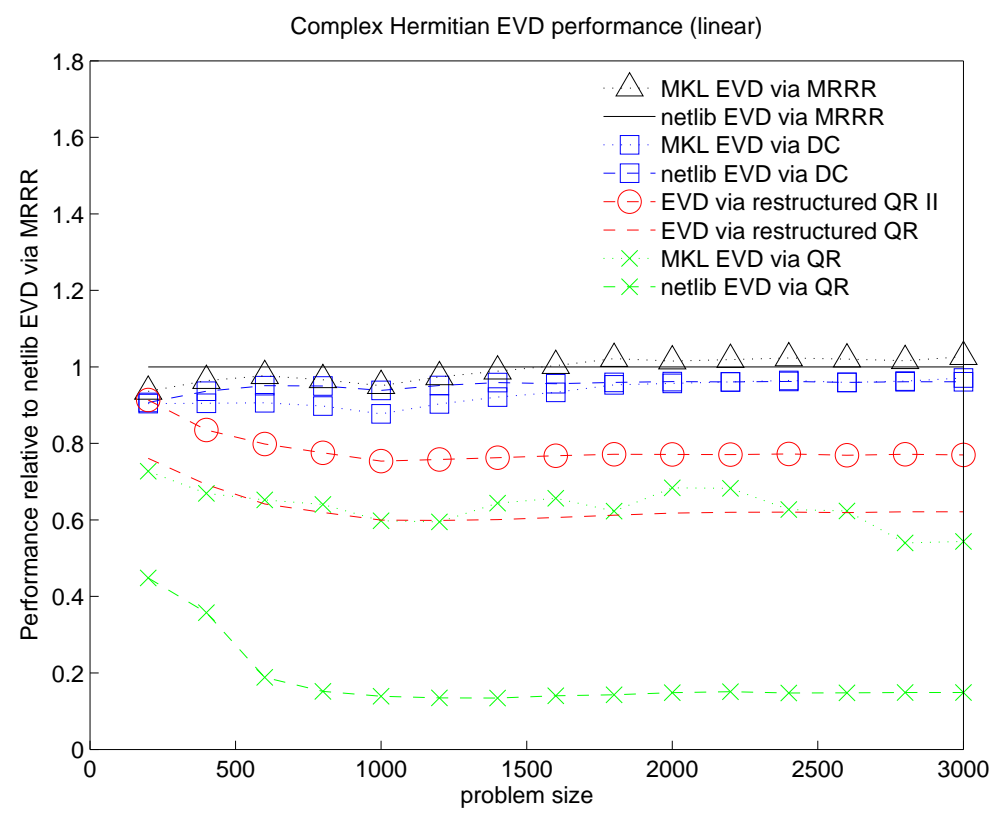

Fig. 17. Performance of implementations relative to netlib EVD via MRRR. Experiments were run with complex matrices generated with linear distributions of eigenvalues.
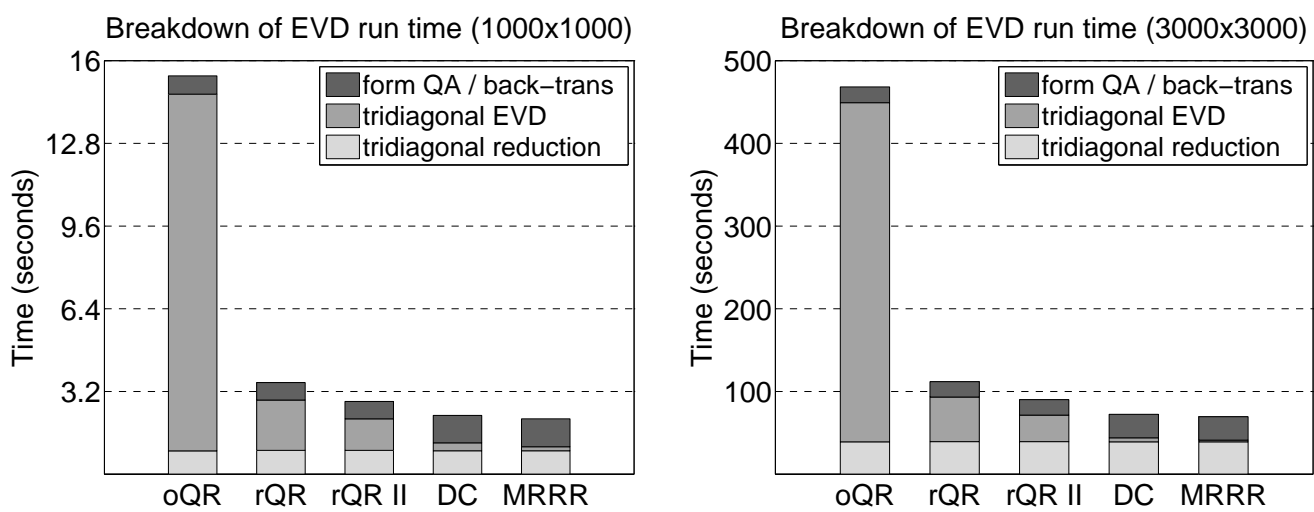

Fig. 18. Breakdown of time spent in the three stages of (complex) Hermitian EVD: tridiagonal reduction, tridiagonal EVD, and the formation of $Q_{A}$ (for QR-based algorithms) or the back-transformation (netlib D\&C and MRRR) for problem sizes of $1000 \times 1000$ (left) and $3000 \times 3000$ (right). Here, "oQR" refers to EVD based on the original netlib QR algorithm while "rQR" refers to our restructured algorithm. This shows how the run time advantage of the tridiagonal D\&C and MRRR algorithms over restructured QR is partially negated by increased time spent in the former methods' back-transformations.

- EVD via restructured QR refers to an EVD based on our restructured tridiagonal QR algorithm linked to OpenBLAS.

- EVD via restructured QR II refers to a variant of EVD via restructured QR that uses the alternative method of forming $Q$ described in Section 6.4.

sizes. When running our tests, we always provided zheevd with additional workspace so that the routine could run at full speed. 
Note that the three EVD implementations found in MKL are widely known to be highly-optimized by experts at Intel. Also, the two EVDs based on our restructured QR algorithm use an implementation of reduction to tridiagonal form that exhibits a performance signature very similar to that of netlib LAPACK's zhetrd.

Default block sizes for netlib LAPACK were used for all component stages of netlib EVD. For each of these suboperations, all of these block sizes happen to be equal to 32. This block size of 32 was also used for the corresponding stages of the EVD via restructured QR. (MKL block sizes are, of course, not known and most likely immutable to the end-user.) For the implementation of APPLYGWAVEBLK, the values $k=32$ and $b=512$ were used. (These parameter values appeared to give somewhat better results than the values that were used when collecting standalone performance results for APPLYGWAVEBLK in Figure 16.)

The results in Figure 17 reveal that using our restructured QR algorithm allows Hermitian EVD performance that is relatively competitive with that of D\&C and MRRR. The variant which uses the alternative method of forming $Q$ performs even more closely with that of D\&C and MRRR.

While compelling, these data report only overall performance; they do not show how each stage of EVD contributes to the speedup. As expected, we found that the time spent in the reduction to tridiagonal form was approximately equal for all netlib implementations and our restructured QR algorithm. ${ }^{11}$ Virtually all of the differences in overall performance were found in the tridiagonal EVD and the back-transformation (or formation of $Q_{A}$ ).

Figure 18 shows, for problem sizes of $1000 \times 1000$ and $3000 \times 3000$, the breakdown of time spent in the reduction to tridiagonal form, the tridiagonal EVD, and the formation of $Q_{A}$ (for the netlib and restructured QR-based algorithms) or the backtransformation (for netlib D\&C and MRRR). It is easy to see why methods based on the original QR algorithm are not commonly used: the tridiagonal EVD is many times more expensive than either of the other stages. This can be attributed to the fact that netlib's zheev applies one set of Givens rotations at a time, and does so via a routine that typically performs on-par with un-optimized level-1 BLAS. These graphs also illustrate why the restructured $\mathrm{QR}$ algorithm is relatively competitive with $\mathrm{D} \& \mathrm{C}$ and MRRR: the run time advantage of using these faster $\mathcal{O}\left(n^{2}\right)$ algorithms is partially offset by an increase in time spent in their back-transformation stages.

The bar graphs in Figure 18 also show that the tridiagonal EVD stage based on the restructured $\mathrm{QR}$ algorithm, in isolation, still significantly underperforms that of D\&C and MRRR. Thus, we reaffirm to the reader that eigenproblems that begin in tridiagonal form should most likely be solved via one of these two latter methods.

\subsection{General singular value decomposition}

We now turn to performance of the SVD of a dense matrix. Figure 19 shows relative performance for six implementations. (Once again, we present results for five other singular value distributions, as well as results for the real domain, in Appendix A.) The implementations tested are:

- MKL SVD via QR refers to MKL zgesvd.

- MKL SVD via D\&C refers to MKL zgesdd.

- netlib SVD via QR refers to netlib zgesvd linked to OpenBLAS.

— netlib SVD via D\&C refers to netlib zgesdd linked to OpenBLAS.

\footnotetext{
${ }^{11}$ Note that we could not confirm this for MKL since we have no way to know that the top-level routineszheev, zheevd, and zheevr-are actually implemented in terms of the three lower-level operations called by the netlib implementation.
} 


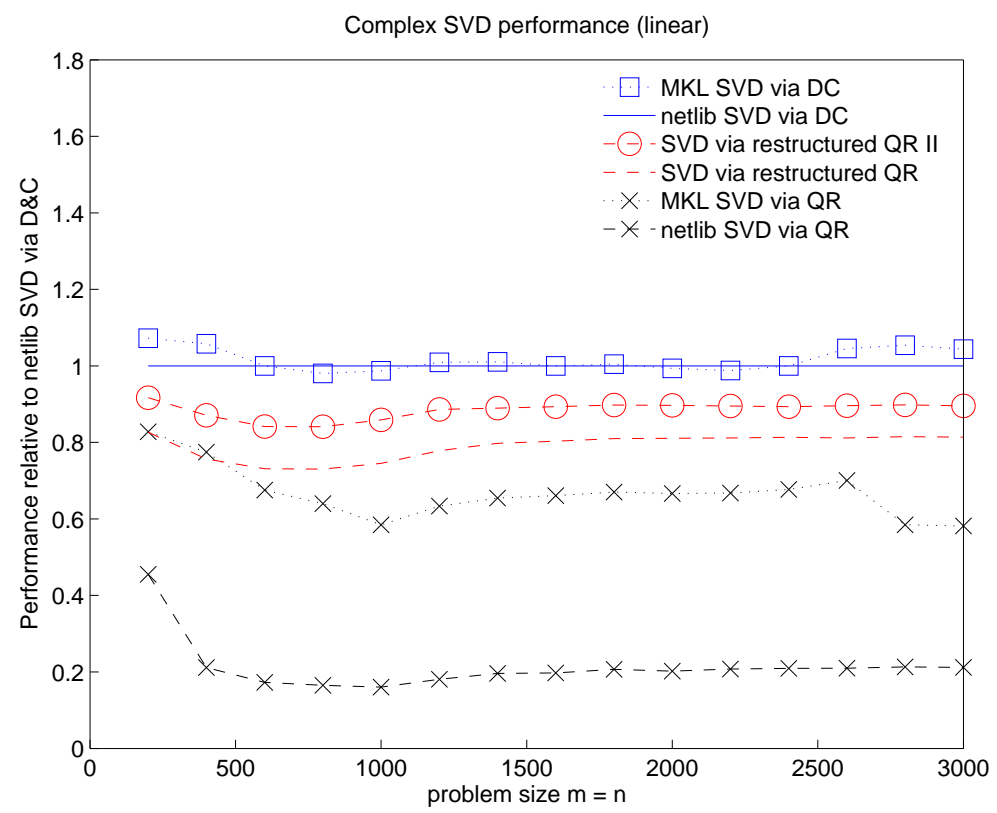

Fig. 19. Performance of implementations relative to netlib SVD via D\&C. Experiments were run with complex matrices generated with linear distributions of singular values. Here, the problem sizes listed are $m=n$. In the case of "SVD via restructured QR" and "SVD via restructured QR II", matrices were reduced to bidiagonal form using a conventional implementation similar to that of zgebrd.
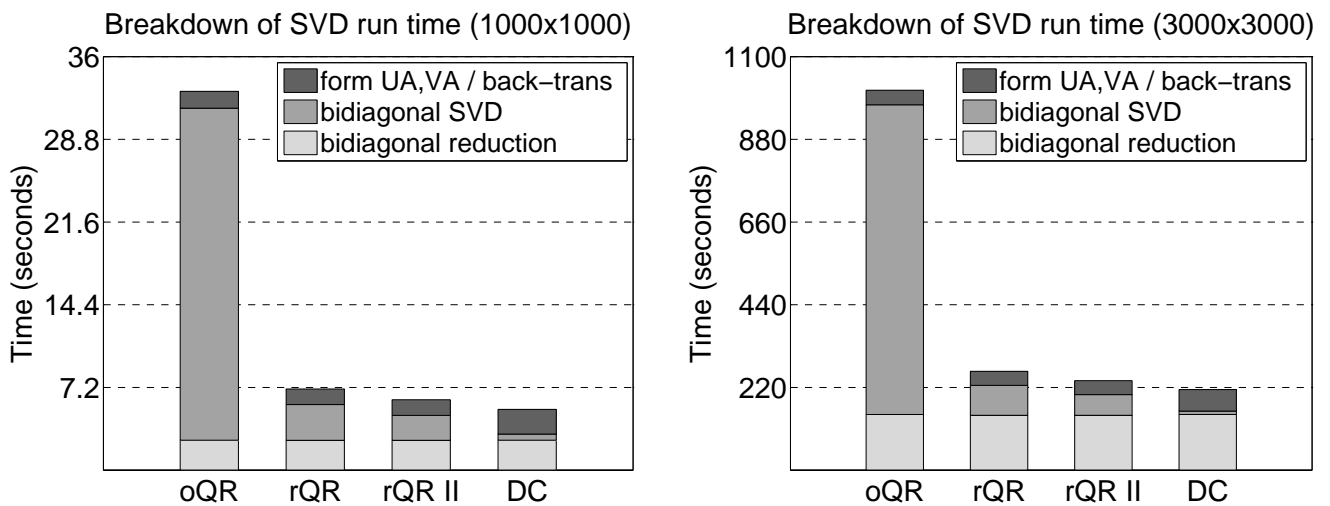

Fig. 20. Breakdown of time spent in the three stages of (complex) SVD: bidiagonal reduction, bidiagonal SVD, and the formation of $U_{A}$ and $V_{A}$ (for QR-based algorithms) or the back-transformation (netlib D\&C) for problem sizes of $1000 \times 1000$ (left) and $3000 \times 3000$ (right). Here, "oQR" refers to EVD based on the original netlib $\mathrm{QR}$ algorithm while "rQR" refers to our restructured algorithm. This shows how the run time advantage of the bidiagonal D\&C algorithm over restructured QR is partially negated by increased time spent in the former method's back-transformations.

- SVD via restructured QR refers to an SVD based on our restructured bidiagonal QR algorithm linked to OpenBLAS.

- SVD via restructured QR II refers to a variant of SVD via restructured $Q R$ that uses an alternative method of forming $U$ and $V$ very similar to that of forming $Q$ in EVD, as described in Section 6.4. 


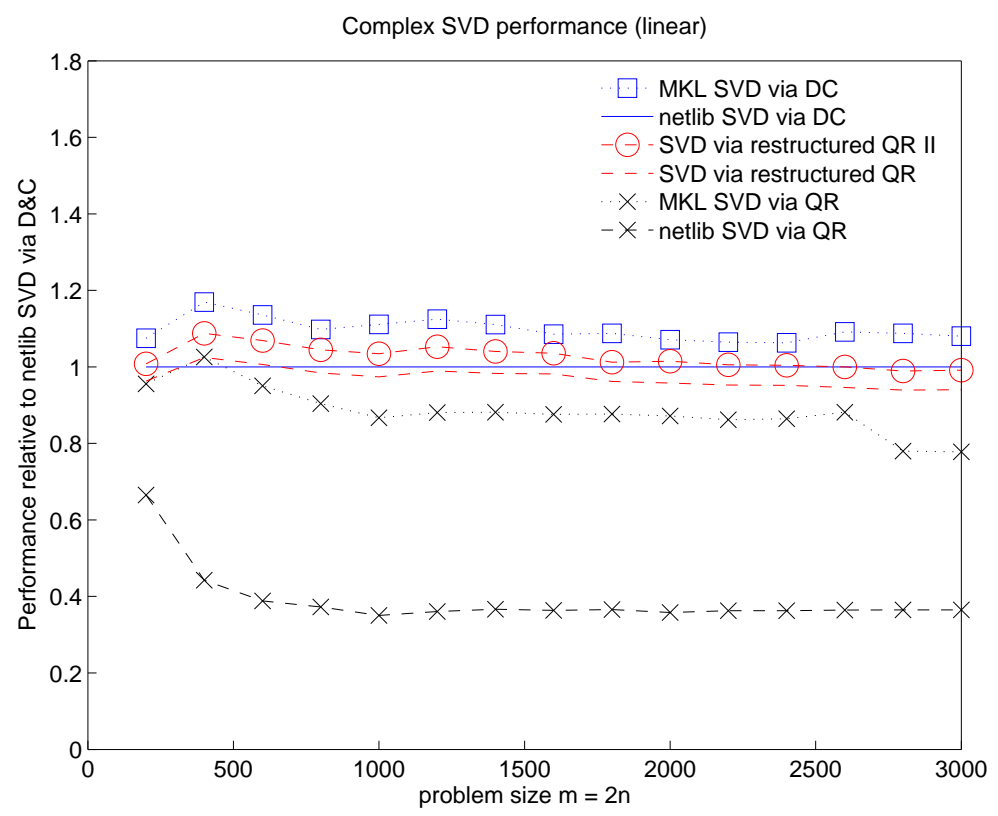

Fig. 21. Performance of implementations relative to netlib SVD via D\&C. Experiments were run with complex matrices generated with linear distributions of singular values. Here, the problem sizes listed are $m=2 n$. In the case of "SVD via restructured QR" and "SVD via restructured QR II", matrices were reduced to bidiagonal form using a conventional implementation similar to that of zgebrd.

Note that we do not compare against an MRRR implementation of SVD since no such implementation currently exists within LAPACK. Also, we use an implementation of reduction to bidiagonal form that performs very similarly to that of netlib LAPACK's zgebrd.

As with the case of EVD, the default block sizes were equal to 32 and also used within the corresponding stages of SVD via restructured QR. Similarly, for APPLYGWAVEBLK, the values $k=32$ and $b=512$ were used.

Looking at the performance results, we once again find that the method based on a restructured $\mathrm{QR}$ algorithm performs very well against a D\&C-based implementation, with the alternate variant performing even better. Figure 20 shows a time breakdown for SVD where $m=n$. The data tell a story similar to that of Figure 18: we find that the benefit to using the D\&C algorithm, when compared to restructured QR, is partially negated by its more costly back-transformation. Figures 21 and 22 show run time and time breakdowns for SVD when the problem size is equal to $n$, where $m=2 n$. These matrices are sufficiently rectangular to trigger the use of a $\mathrm{QR}$ factorization, as described in Section 8. (We know the netlib codes, as well as our implementation using restructured $\mathrm{QR}$, use the $\mathrm{QR}$ factorization for these problem shapes, though we are unsure of MKL since it is a proprietary product.) Aside from the additional step of performing the $\mathrm{QR}$ factorization, it also requires a corresponding additional back-transformation, performed via zgemm, to multiply the unitary matrix $Q$ by the intermediate product $U_{R} U_{B}$. However, the time spent in both the $\mathrm{QR}$ factorization and the $Q$ back-transformations is relatively small, and very consistent across methods.

The results show that this $m=2 n$ problem size configuration actually allows the restructured QR-based SVD to match D\&C's performance. Figure 22 reveals why: the 

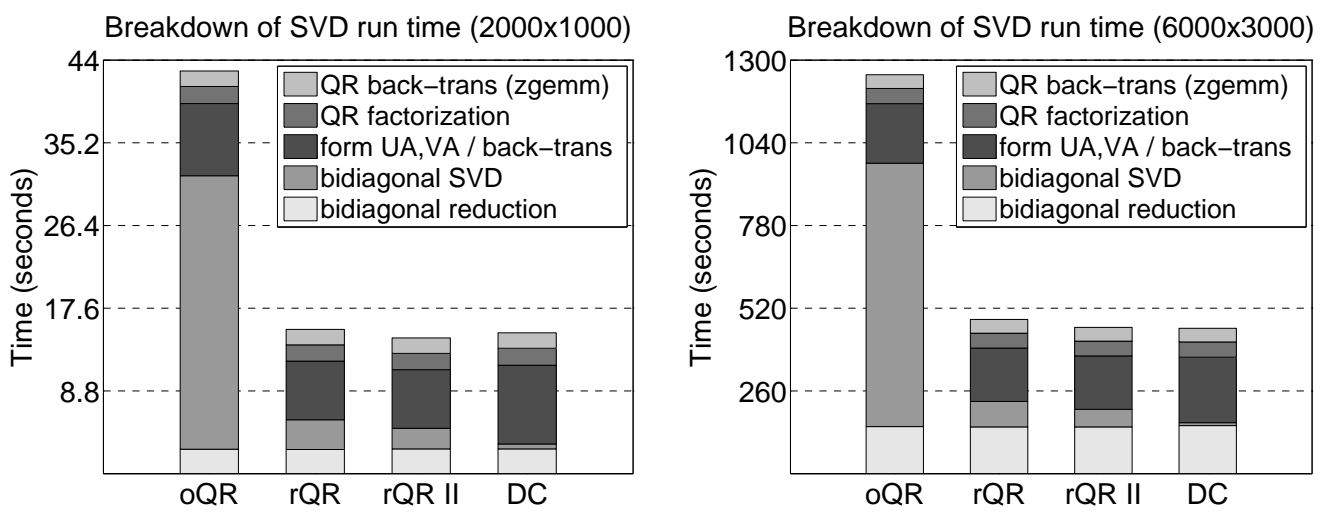

Fig. 22. Breakdown of time spent in the three stages of (complex) SVD: bidiagonal reduction, bidiagonal SVD, and the formation of $U_{A}$ and $V_{A}$ (for QR-based algorithms) or the back-transformation (netlib D\&C) for problem sizes of $2000 \times 1000$ (left) and $6000 \times 3000$ (right). Here, "oQR" refers to EVD based on the original netlib QR algorithm while "rQR" refers to our restructured algorithm. In contrast to Figure 20, these graphs reflect the additional time spent in the $\mathrm{QR}$ factorization of $A$ and the corresponding backtransformation necessary to combine $Q$ with $U_{R} U_{B}$, which is implemented via zgemm.

extra time spent forming $Q$, along with back-transforming $U_{A}$ with $U_{B}$ (and $V_{A}$ with $V_{B}$ ) virtually erases the time saved by using D\&C for the bidiagonal SVD.

As with the tridiagonal EVD stage of dense Hermitian EVD, we can intuit from Figures 20 and 22 that D\&C-based SVD would continue to offer superior performance over the restructured $\mathrm{QR}$ algorithm when the input matrix happens to already be in bidiagonal form.

Now that there exist high-performance implementations of both the QR and D\&C algorithms (and their back-transformations or equivalent stages) the last remaining performance bottleneck is the reduction to bidiagonal form. However, there is potential for speedup via this operation, too. The authors of [Van Zee et al. 2012], building on the efforts of [Howell et al. 2008], report on an implementation of reduction to bidiagonal form that is $60 \%$ faster, asymptotically, than the reference implementation provided by netlib LAPACK. For cases where $m=n$, we found the bidiagonal reduction to constitute anywhere from 40 to $60 \%$ of the total SVD run time when using the restructured QR algorithm. Thus, combining the results of that paper with this one would further accelerate the overall SVD. (The impact of this faster reduction on the overall SVD can be seen in the curves labeled "libflame SVD (fast BiRed + QR)" and "libflame SVD (fast BiRed + QR II)" in Figures 29-34 of Appendix A.) While, admittedly, this bidiagonal reduction could be used to speed up any code based on bidiagonal SVD, the libflame library is the only dense linear algebra library we know of to currently offer such an implementation.

\section{CONCLUSIONS}

Ever since the inception of the level-3 BLAS, some have questioned whether a routine for applying multiple sets of Givens rotations should have been part of the BLAS. We believe that the present paper provides compelling evidence that the answer is in the affirmative.

One could render quick judgment on this work by pointing out that the restructured $\mathrm{QR}$ algorithm facilitates performance that is merely competitive with, not faster than, that of a number algorithms that were developed in the 1980s and 1990s. But one could argue that there is undervalued virtue in simplicity and reliability (and the QR algorithm exhibits both), especially if it comes at the expense of only minor performance 
degradation. And to some, the option of $\mathcal{O}(n)$ workspace is important. The $\mathrm{D} \& \mathrm{C}$ and MRRR algorithms took many years to tweak and perfect (mostly for accuracy reasons) and require an expert to maintain and extend. By contrast, the $\mathrm{QR}$ algorithm as we present it is essentially identical to the one developed for EISPACK [Smith et al. 1976] in the 1960s, which can be understood and mastered by a typical first-year graduate student in numerical linear algebra. Perhaps most importantly, the QR algorithm's numerical robustness is undisputed, and now that its performance (in the case of dense matrices) is competitive, we would argue that it should be preferred over other alternatives because it can reliably solve a wide range of problems.

One important caveat to this work is that we show the restructured QR algorithm to be competitive with other methods only in the context of Hermitian EVD and SVD on dense matrices (and only when all eigen-/singular vectors are required). While the restructuring and optimizations discussed here would greatly benefit someone who had decided $a$ priori to use the $\mathrm{QR}$ algorithm when the input matrix is already tridiagonal or bidiagonal, such individuals are most likely best served by D\&C or MRRR, as those standalone methods still offer substantially shorter runtimes.

The results presented in this paper present several avenues for future research.

- We believe that the application of Givens rotations within the restructured QR algorithm may be parallelized for multicore systems with relatively minor changes. Acceleration with GPUs should also be possible. In some sense, limiting our discussion in this paper to only a single core favors other methods: on multiple cores, the reduction to tridiagonal form will, relatively speaking, become even more of an expense since it is largely limited by bandwidth to main memory. Recent re-emergence of successive band reduction tries to overcome this [Luszczek et al. 2011; Bientinesi et al. 2011; Haidar et al. 2011].

- Now that the QR algorithm is competitive with more recent algorithms, it may be worth attempting to improve the shifting mechanism. For example, in [Jiang and Zhang 1985] it is shown that a hybrid method that uses both Rayleigh Quotient shifts and Wilkinson shifts is cubically (and globally) convergent. This may reduce the number of Francis steps for many matrix types, which would decrease the overall runtime of the QR algorithm. Alternatively, in [Dhillon 1997] it is suggested that, if eigenvalues are computed first, a "perfect shift" can be incorporated in the QR algorithm that causes deflation after only one iteration, though the same document uncovers certain challenges associated with this approach.

- "Aggressive early deflation" has been developed to accelerate the QR algorithm for upper Hessenberg matrices [Braman et al. 2001b], and the authors of [Nakatsukasa et al. 2012] have extended the approach to the $d q d s$ algorithm. Thus, it may be possible to incorporate this technique into our restructured $\mathrm{QR}$ algorithm.

- Previous efforts have succeeded in achieving level-3 BLAS performance in the aforementioned Hessenberg QR algorithm by applying multiple shifts and chasing multiple bulges simultaneously [Braman et al. 2001a]. It may be possible to further improve those methods by restructuring the computation so that the wavefront algorithm for applying Givens rotations may be employed.

- Periodically, the Jacobi iteration (for the EVD and SVD) receives renewed attention [Jacobi 1846; Demmel and Veselić 1992; Drmač 2009; Drmač and Veselić 2008a; 2008b]. Since, like the QR algorithm, Jacobi-based approachs rely on Givens rotations, this work may further benefit those methods. Now that the excellent performance of a routine that applies sets of Givens rotations has been demonstrated, an interesting question is how to incorporate this into a blocked Jacobi iteration.

Thus, our work may result in a renaissance for the QR algorithm. 


\section{Acknowledgments}

We kindly thank G. W. (Pete) Stewart, Paolo Bientinesi, Jack Poulson, and Daniel Kressner for offering valuable advice, expertise, and feedback on a working draft of the paper.

This research was partially sponsored by the UTAustin-Portugal Colab program, a grant from Microsoft, and grants from the National Science Foundation (Awards OCI0850750, CCF-0917167, and OCI-1148125).

Any opinions, findings and conclusions or recommendations expressed in this material are those of the author(s) and do not necessarily reflect the views of the National Science Foundation (NSF).

\section{REFERENCES}

Anderson, E., Bai, Z., Bischof, C., Blackford, L. S., Demmel, J., Dongarra, J. J., Croz, J. D., Hammarling, S., Greenbaum, A., McKenney, A., And Sorensen, D. 1999. LAPACK Users' Guide (third ed.). Society for Industrial and Applied Mathematics, Philadelphia, PA, USA.

Bientinesi, P., Igual, F. D., Kressner, D., Petschow, M., And Quintana-Ortí, E. S. 2011. Condensed forms for the symmetric eigenvalue problem on multi-threaded architectures. Concurr. Comput. : Pract. Exper. 23, 7, 694-707.

Bischof, C., LANG, B., AND SUn, X. 1994. Parallel tridiagonalization through two-step band reduction. In In Proceedings of the Scalable High-Performance Computing Conference. IEEE Computer Society Press, 23-27.

BISCHOF, C. AND VAN LOAN, C. 1987. The WY representation for products of Householder matrices. SIAM J. Sci. Stat. Comput. 8, 1, 2-13.

BLAST 2002. Basic linear algebra subprograms technical forum standard. International Journal of High Performance Applications and Supercomputing 16, 1.

Braman, K., Byers, R., AND MathiAs, R. 2001a. The multishift $Q R$ algorithm. part I: Maintaining wellfocused shifts and level 3 performance. SIAM J. Matrix Anal. Appl. 23, 929-947.

BRAmAn, K., BYERS, R., AND MATHIAS, R. 2001b. The multishift $Q R$ algorithm. part II: Aggressive early deflation. SIAM J. Matrix Anal. Appl. 23, 948-973.

Cline, A. K. AND Dhillon, I. S. 2006. Handbook of Linear Algebra. CRC Press, Chapter Computation of the Singular Value Decomposition, 45-1-45-13.

Cuppen, J. J. M. 1980. A divide and conquer method for the symmetric tridiagonal eigenproblem. $\mathrm{Nu}$ merische Mathematik 36, 177-195.

Demmel, J., Dhillon, I., AND REN, H. 1995. On the correctness of some bisection-like parallel eigenvalue algorithms in floating point arithmetic. Electronic Trans. Num. Anal 3, 116-149.

Demmel, J. AND KAhAN, W. 1990. Accurate singular values of bidiagonal matrices. SIAM J. Sci. Stat. Comput. 11, 873-912.

Demmel, J., Marques, O., PARlett, B., ANd Vömel, C. 2008. Performance and accuracy of LAPACK's symmetric tridiagonal eigensolvers. SIAM J. Sci. Comput. 30, 3, 1508-1526.

Demmel, J. AND VeSELIĆ, K. 1992. Jacobi's method is more accurate than QR. SIAM Journal on Matrix Analysis and Applications 13, 4, 1204-1245.

DHILloN, I. S. 1997. A new $O\left(n^{2}\right)$ algorithm for the symmetric tridiagonal eigenvalue/eigenvector problem. Ph.D. thesis, EECS Department, University of California, Berkeley.

Dhillon, I. S. 1998. Current inverse iteration software can fail. BIT Numer. Math. 38, 685-704.

Dhillon, I. S. AND PARLETT, B. N. 2003. Orthogonal eigenvectors and relative gaps. SIAM J. Matrix Anal. Appl. 25, 3, 858-899.

Dhillon, I. S. AND PARLeTt, B. N. 2004. Multiple representations to compute orthogonal eigenvectors of symmetric tridiagonal matrices. Lin. Alg. Appl. 387, 1-28.

Dhillon, I. S., PARlett, B. N., AND Vömel, C. 2006. The design and implementation of the MRRR algorithm. ACM Trans. Math. Soft. 32, 533-560.

Dongarra, J. J., Du Croz, J., Hammarling, S., And Hanson, R. J. 1988. An extended set of FORTRAN basic linear algebra subprograms. ACM Trans. Math. Soft. 14, 1, 1-17.

DRMAČ, Z. 2009. A global convergence proof for cyclic Jacobi methods with block rotations. SIAM J. Matrix Anal. Appl. 31, 3, 1329-1350.

DrmaČ, Z. AND VESELIĆ, K. 2008a. New fast and accurate Jacobi SVD algorithm. I. SIAM J. Matrix Anal. Appl. 29, 4, 1322-1342. 
DRMAČ, Z. AND VESELIĆ, K. 2008b. New fast and accurate Jacobi SVD algorithm. II. SIAM J. Matrix Anal. Appl. 29, 4, 1343-1362.

Fernando, K. V. AND PARLett, B. N. 1994. Accurate singular values and differential qd algorithms. Numer. Math. 67, 191-229.

Golub, G. H. And LoAn, C. F. V. 1996. Matrix Computations 3rd Ed. The Johns Hopkins University Press, Baltimore.

Goto, K. AND VAN DE Geijn, R. A. 2008. Anatomy of a high-performance matrix multiplication. ACM Trans. Math. Soft. 34, 3.

GU, M. AND Eisenstat, S. C. 1995a. A divide-and-conquer algorithm for the bidiagonal SVD. SIAM J. Matrix Anal. Appl. 16, 1, 79-92.

Gu, M. And Eisenstat, S. C. 1995b. A divide-and-conquer algorithm for the symmetric tridiagonal eigenproblem. SIAM J. Matrix Anal. Appl. 16, 1, 172-191.

Haidar, A., Ltaief, H., AND DongarRa, J. 2011. Parallel reduction to condensed forms for symmetric eigenvalue problems using aggregated fine-grained and memory-aware kernels. LAPACK Working Note 254. August.

Howell, G. W., Demmel, J. W., Fulton, C. T., Hammarling, S., AND Marmol, K. 2008. Cache efficient bidiagonalization using BLAS 2.5 operators. ACM Transactions on Mathematical Software 34, 3, 14:114:33.

JACOBI, C. 1846. Über ein leichtes verfahren, die in der theorie der säkularstörungen vorkommenden gleichungen numerisch aufzulösen. Crelle's Journal 30, 51-94.

JIANG, E. AND ZHANG, Z. 1985. A new shift of the QL algorithm for irreducible symmetric tridiagonal matrices. Linear Algebra and its Applications 65, 0, 261-272.

Joffrain, T., Low, T. M., QuintanA-Ortí, E. S., VAN DE GEIJn, R., AND VAN ZeE, F. 2006. Accumulating Householder transformations, revisited. ACM Transactions on Mathematical Software 32, 2, 169-179.

KÅGström, B., Kressner, D., Quintana-Ortí, E. S., And Quintana-Ortí, G. 2008. Blocked algorithms for the reduction to Hessenberg-triangular form revisited. BIT Numer. Math. 48, 3, 563-584.

LANG, B. 1998. Using level 3 BLAS in rotation-based algorithms. SIAM J. Sci. Comput. 19, 2, 626-634.

LAPACK. 2011. http://www.netlib.org/lapack/.

LAwson, C. L., HANson, R. J., KinCAID, D. R., AND KROGH, F. T. 1979. Basic linear algebra subprograms for Fortran usage. ACM Trans. Math. Soft. 5, 3, 308-323.

LIBFLAME. 2011. http://www.cs.utexas . edu/users/flame/libflame/.

Luszczek, P., LtAief, H., AND DongarRA, J. 2011. Two-stage tridiagonal reduction for dense symmetric matrices using tile algorithms on multicore architectures. LAPACK Working Note 244. April.

NAKATsukAsA, Y., Aishima, K., AND YAMAZAKI, I. 2012. dqds with aggressive early deflation. SIAM J. Matrix Anal. Appl. 33, 1, 22-51.

OPENBLAS. 2011. https://github.com/xianyi/OpenBLAS/.

Parlett, B. N. 1980. The Symmetric Eigenvalue Problem. Prentice-Hall.

Parlett, B. N. ANd Marques, O. A. 1999. An implementation of the dqds algorithm (positive case). Lin. Alg. Appl. 309, 217-259.

QUINTANA-ORTÍ, G. AND VIDAL, A. M. 1992. Parallel algorithms for QR factorization on shared memory multiprocessors. In European Workshop on Parallel Computing'92, Parallel Computing: From Theory to Sound Practice. IOS Press, 72-75.

RAJAMANICKAM, S. 2009. Efficient algorithms for sparse singular value decomposition. Ph.D. thesis, University of Florida.

RUtTER, J. D. 1994. A serial implementation of Cuppen's divide and conquer algorithm for the symmetric eigenvalue problem. Tech. Rep. CSD-94-799, Computer Science Division, University of California at Berkeley. February.

SCHREIBER, R. AND VAN LOAN, C. 1989. A storage-efficient WY representation for products of Householder transformations. SIAM J. Sci. Stat. Comput. 10, 1, 53-57.

Smith, B. T. ET AL. 1976. Matrix Eigensystem Routines - EISPACK Guide Second Ed. Lecture Notes in Computer Science 6. Springer-Verlag, New York.

Stewart, G. W. 2001. Matrix Algorithms Volume II: Eigensystems. Society for Industrial and Applied Mathematics, Philadelphia, PA, USA.

VAN ZEE, F. G. 2011. libflame: The Complete Reference. http://www.lulu.com/.

VAN ZeE, F. G., VAN DE GEIJN, R., AND QuintanA-ORTÍ, G. 2011. Restructuring the QR algorithm for high-performance application of Givens rotations. Tech. Rep. TR-11-36, The University of Texas at Austin, Department of Computer Science. October. FLAME Working Note \#60. 
Van Zee, F. G., VAn De Geijn, R. A., Quintana-Ortí, G., And Elizondo, G. J. 2012. Families of algorithms for reducing a matrix to condensed form. ACM Trans. Math. Soft. 39, 1, xxx-yyy.

WATKINS, D. S. 1982. Understanding the $Q R$ algorithm. SIAM Review 24, 4, 427-440.

WILKInson, J. H. 1968. Global convergence of tridiagonal $Q R$ algorithm with origin shifts. Linear Algebra and its Applications 1, 409-420.

Willems, P. R. AND LANG, B. 2011. A framework for the $\mathrm{MR}^{3}$ algorithm: Theory and implementation. Tech. rep., Bergische Universität Wuppertal. April.

Willems, P. R., LANG, B., AND VÖMEL, C. 2006. Computing the bidiagonal SVD using multiple relatively robust representations. SIAM J. Matrix Anal. Appl. 28, 4, 907-926. 


\section{Online Appendix to: Restructuring the Tridiagonal and Bidiagonal QR Algorithms for Performance}

FIELD G. VAN ZEE, The University of Texas at Austin

ROBERT A. VAN DE GEIJN, The University of Texas at Austin

GREGORIO QUINTANA-ORTÍ, Universitat Jaume I

\section{A. ADDITIONAL PERFORMANCE RESULTS}

Authors' note: We envision this appendix being published through ACM TOMS as an electronic appendix.

In this section, we present complete results for six eigen-/singular value distributions for Hermitian EVD as well as SVD where $m=n$. The distribution types for eigen-/singular values $\lambda_{i}$, for $i \in 0,1,2, \ldots, n-1$, are a follows:

- Geometric distributions are computed as $\lambda_{i}=\alpha(1-\alpha)^{i+1}$. For our experiments, we used $\alpha=\frac{1}{n}$.

- Inverse distributions are computed as $\lambda_{i}=\frac{\alpha}{i+1}$. For our experiments, we used $\alpha=1$.

— Linear distributions are computed as $\lambda_{i}=\beta+\alpha(i+1)$. For our experiments, we used $\alpha=1$ and $\beta=0$.

- Logarithmic distributions are computed as $\lambda_{i}=\frac{\alpha^{i+1}}{\alpha^{n}}$. For our experiments, we used $\alpha=1.2$.

- Random distributions are computed randomly over an interval $[\sigma, \sigma+\omega]$. For our experiments, we used $\sigma=0$ and $\omega=n$.

- Cluster distributions are generated containing $n_{c}$ clusters, roughly equally-sized, where half of the overall spectrum lies in one of the $n_{c}$ clusters while the remaining half resides in the (relatively) sparsely populated region between clusters. More specifically, this spectrum is generated by alternating between linear and random distributions. The first segment of the distribution is linear and roughly $\frac{n}{2 n_{c}}$ values are generated (with $\beta$ initially zero, and $\alpha=1$ which remains constant), up to some $\lambda_{j}$. This value $\lambda_{j}$ then becomes $\sigma$ in a random distribution, where $0<\omega \ll 1$. The last value in the random interval serves as the $\beta$ for the next linear segment, and so forth until $2 n_{c}$ separate regions have been created. For our experiments, we used $n_{c}=10$ and $\omega=10^{-9}$.

(c) 2012 ACM 0098-3500/2012/01-ART00 $\$ 10.00$

DOI 10.1145/0000000.0000000 http://doi.acm.org/10.1145/0000000.0000000 

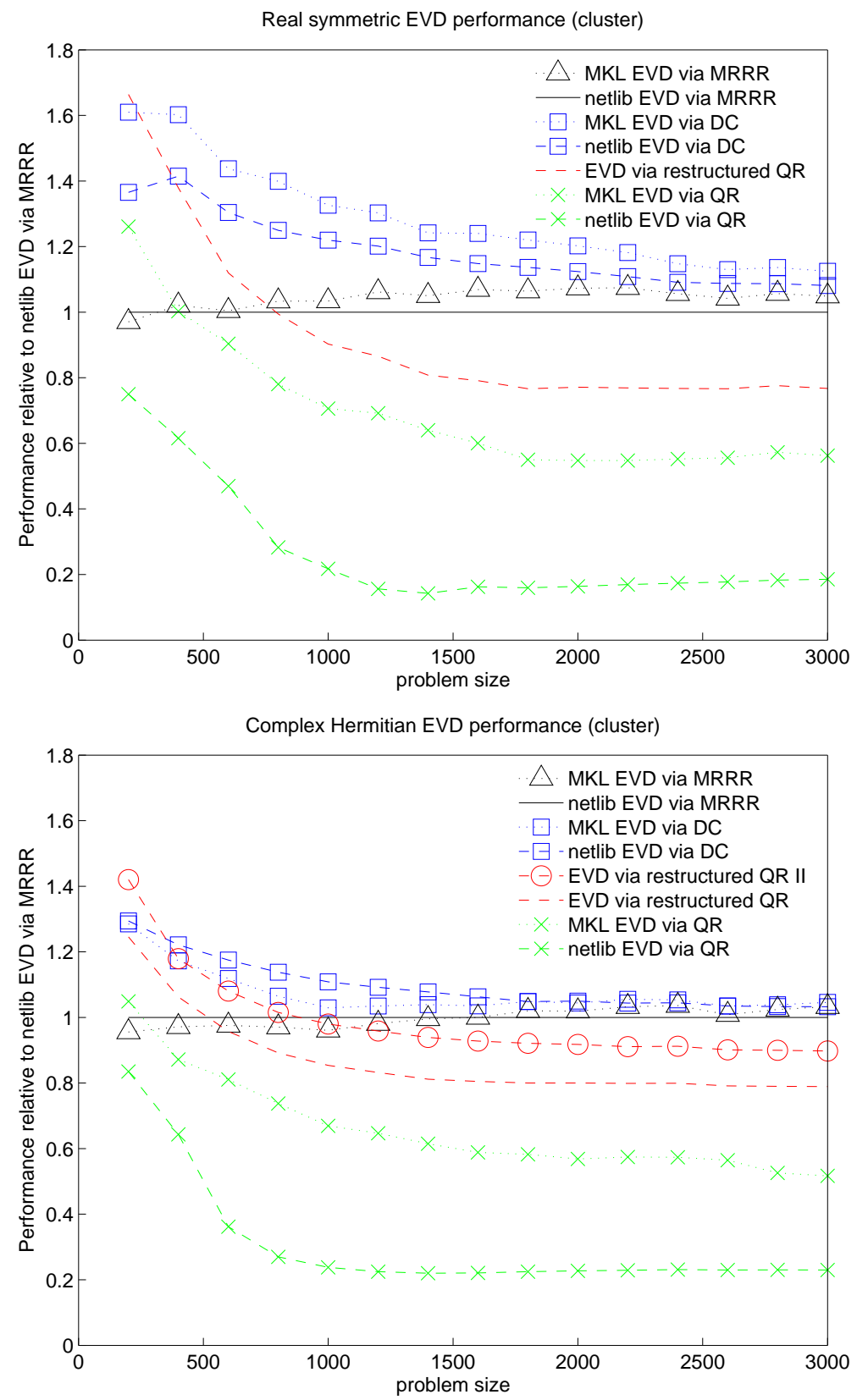

Fig. 23. Performance of implementations relative to netlib EVD via MRRR for real (top) and complex (bottom) matrices generated with clustered distributions of eigenvalues. In the case of EVD via restructured QR, matrices were reduced to tridiagonal form using an implementation that exhibits a performance signature nearly identical to that of dsytrd/zhetrd. 

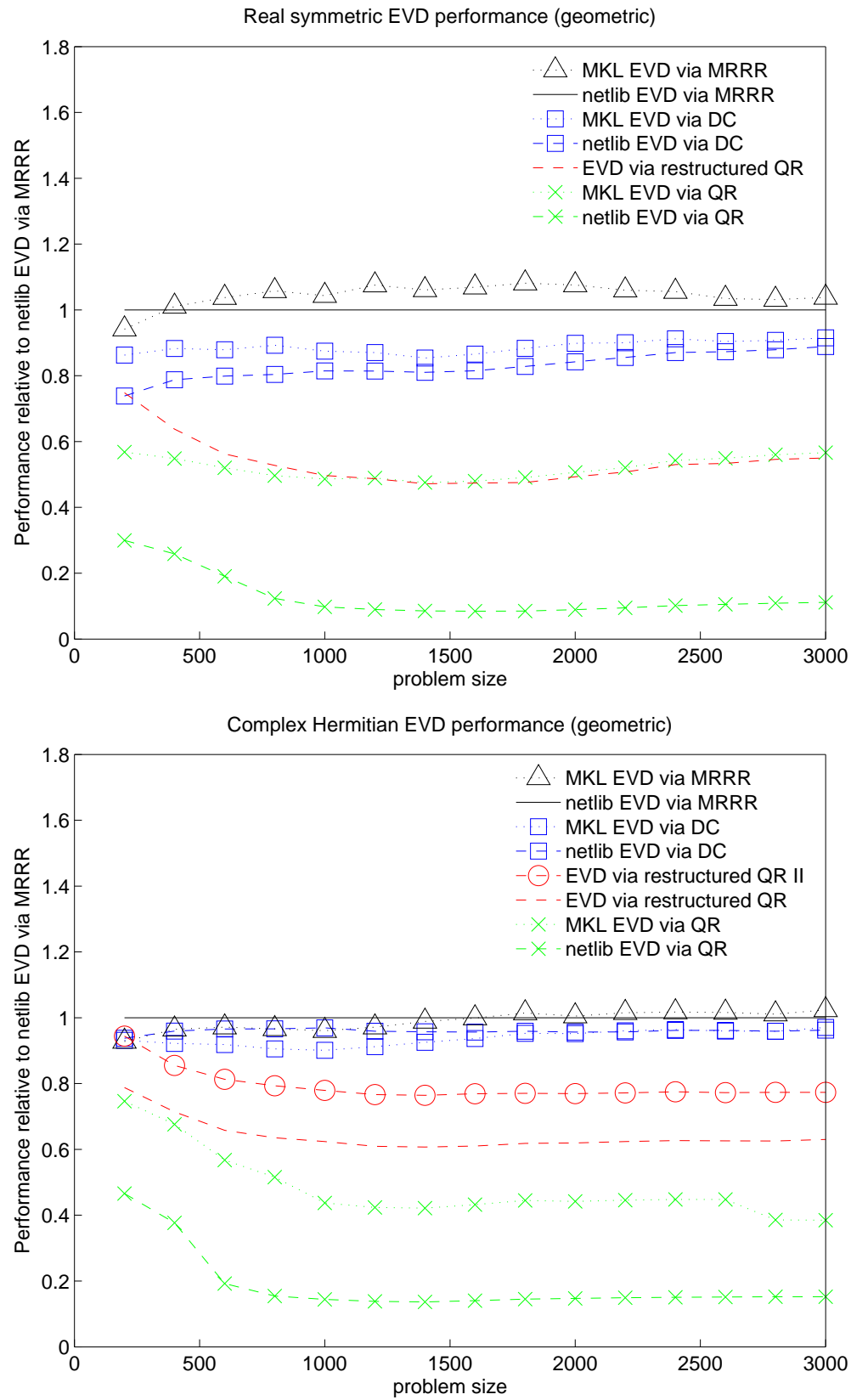

Fig. 24. Performance of implementations relative to netlib EVD via MRRR for real (top) and complex (bottom) matrices generated with geometric distributions of eigenvalues. In the case of EVD via restructured $\mathrm{QR}$, matrices were reduced to tridiagonal form using an implementation that exhibits a performance signature nearly identical to that of dsytrd/zhetrd. 

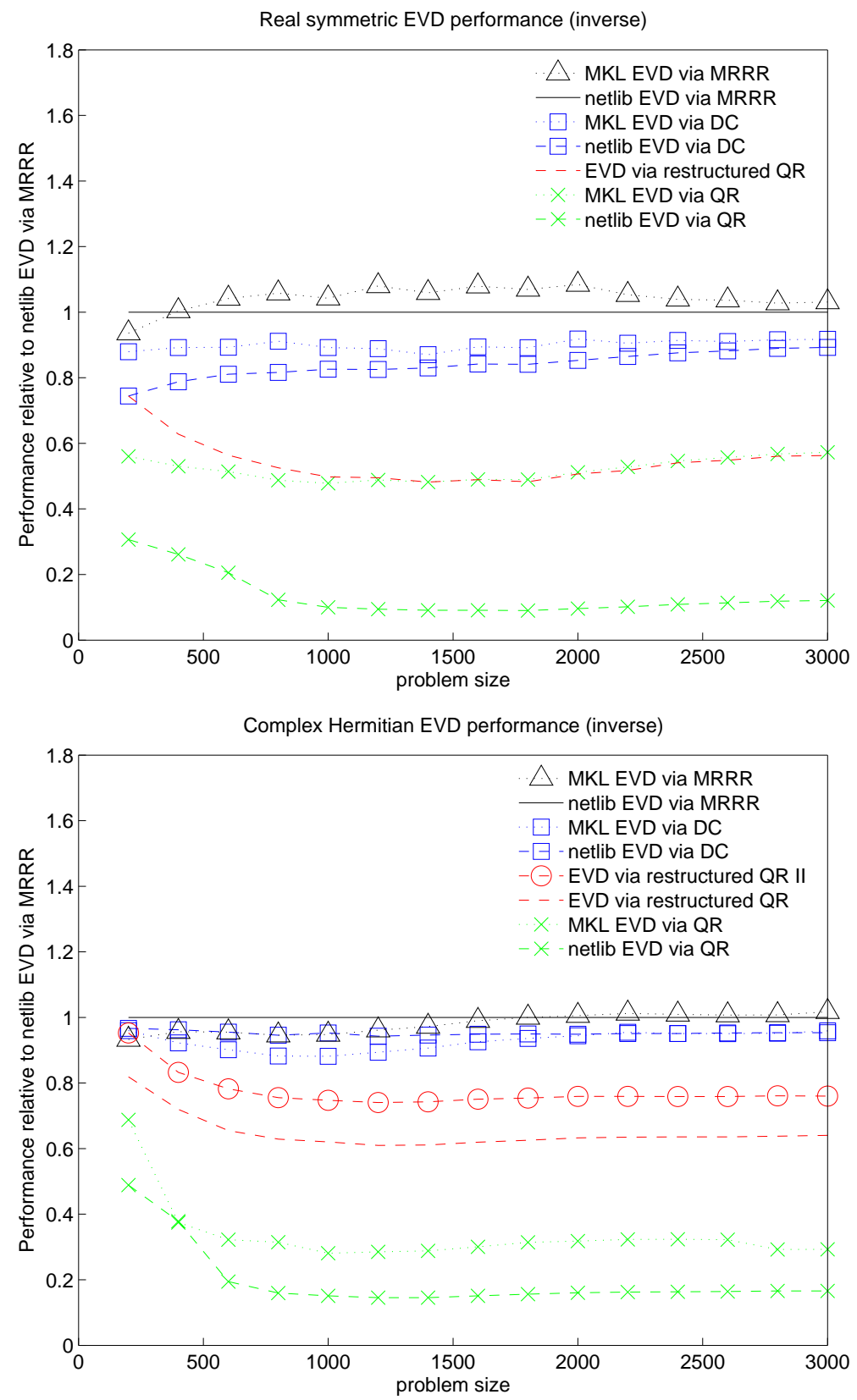

Fig. 25. Performance of implementations relative to netlib EVD via MRRR for real (top) and complex (bottom) matrices generated with inverse distributions of eigenvalues. In the case of EVD via restructured QR, matrices were reduced to tridiagonal form using an implementation that exhibits a performance signature nearly identical to that of dsytrd/zhetrd. 

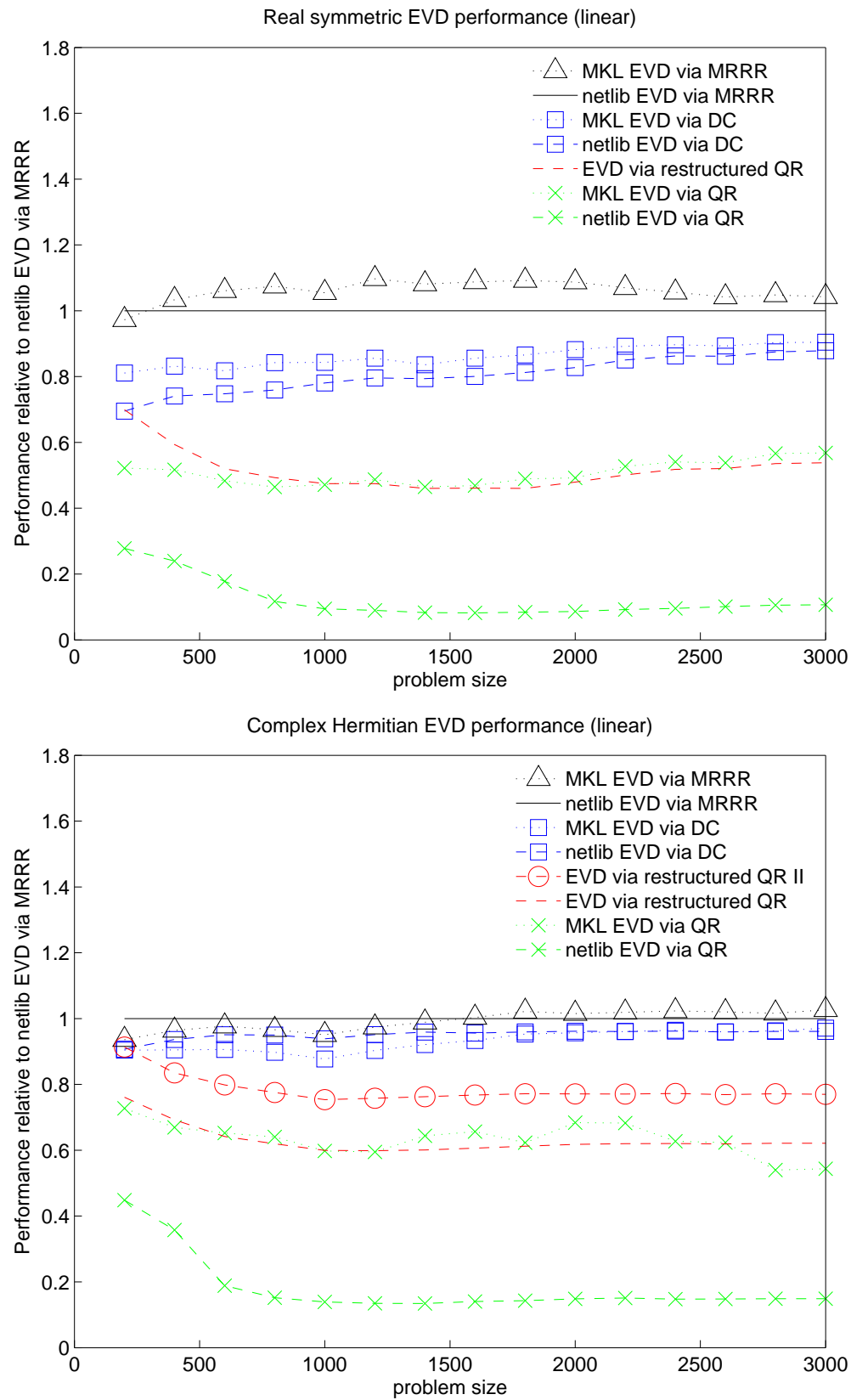

Fig. 26. Performance of implementations relative to netlib EVD via MRRR for real (top) and complex (bottom) matrices generated with linear distributions of eigenvalues. In the case of EVD via restructured QR, matrices were reduced to tridiagonal form using an implementation that exhibits a performance signature nearly identical to that of dsytrd/zhetrd. 

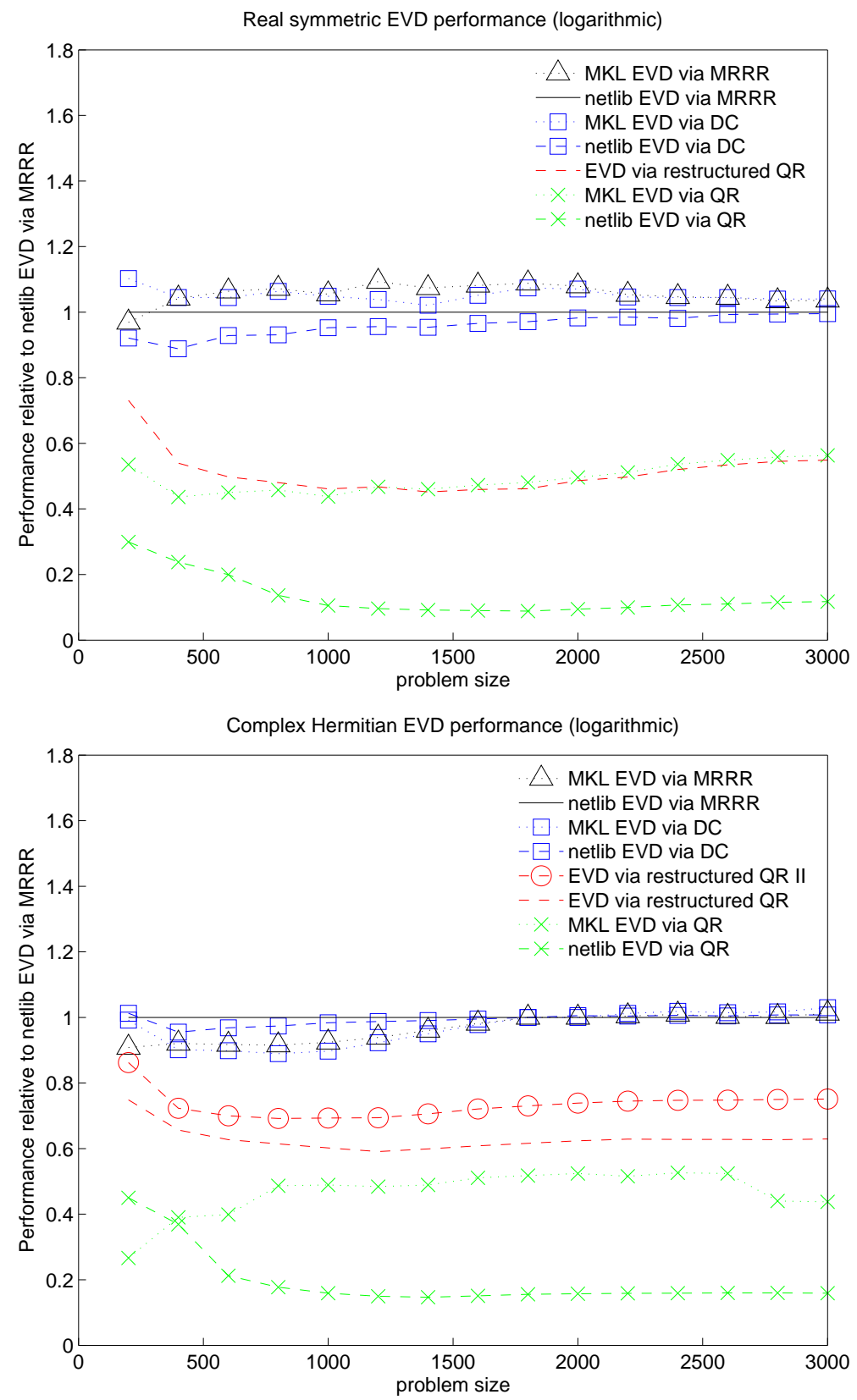

Fig. 27. Performance of implementations relative to netlib EVD via MRRR for real (top) and complex (bottom) matrices generated with logarithmic distributions of eigenvalues. In the case of EVD via restructured $\mathrm{QR}$, matrices were reduced to tridiagonal form using an implementation that exhibits a performance signature nearly identical to that of dsytrd/zhetrd. 

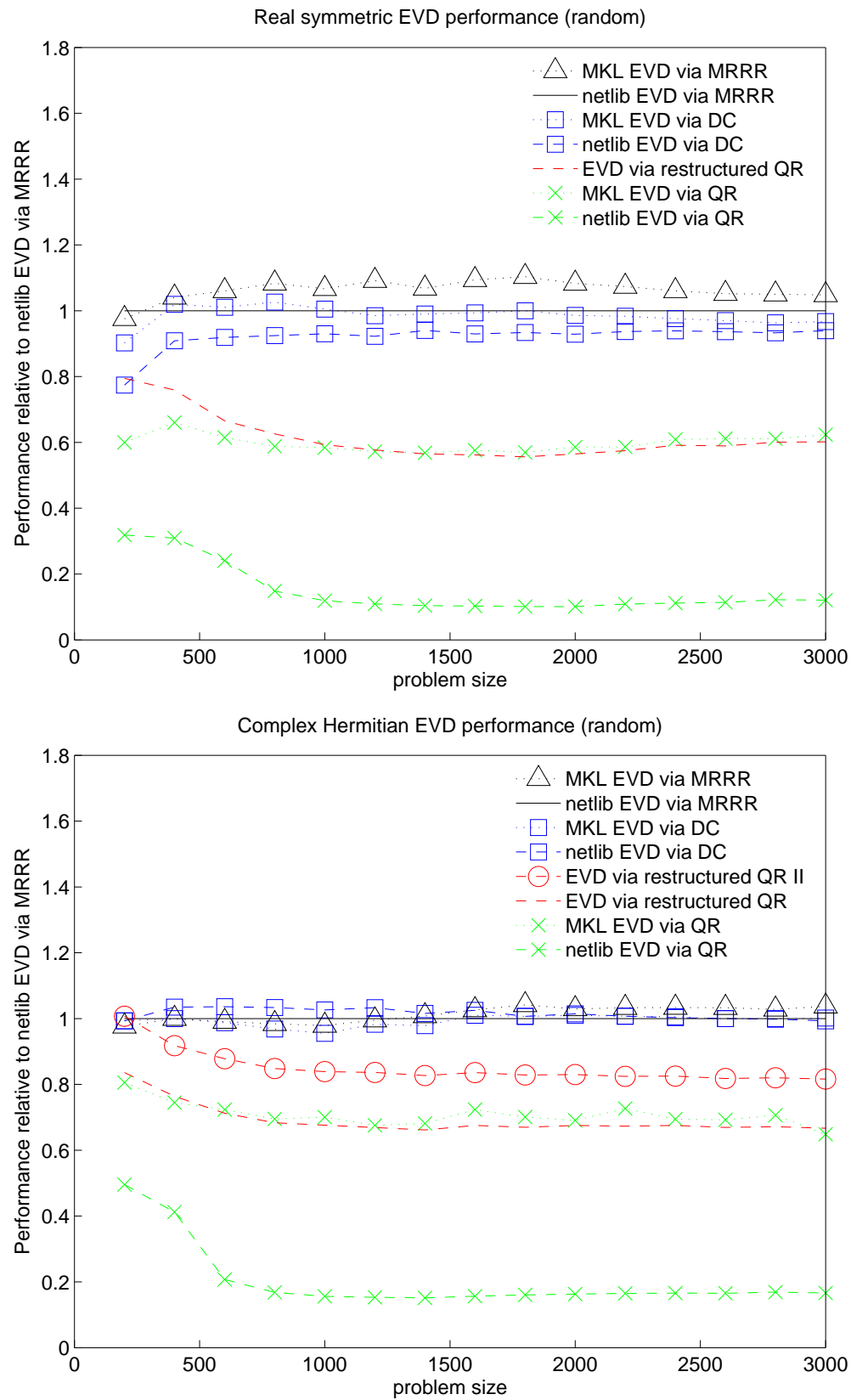

Fig. 28. Performance of implementations relative to netlib EVD via MRRR for real (top) and complex (bottom) matrices generated with random distributions of eigenvalues. In the case of EVD via restructured QR, matrices were reduced to tridiagonal form using an implementation that exhibits a performance signature nearly identical to that of dsytrd/zhetrd. 
Real SVD performance (cluster)

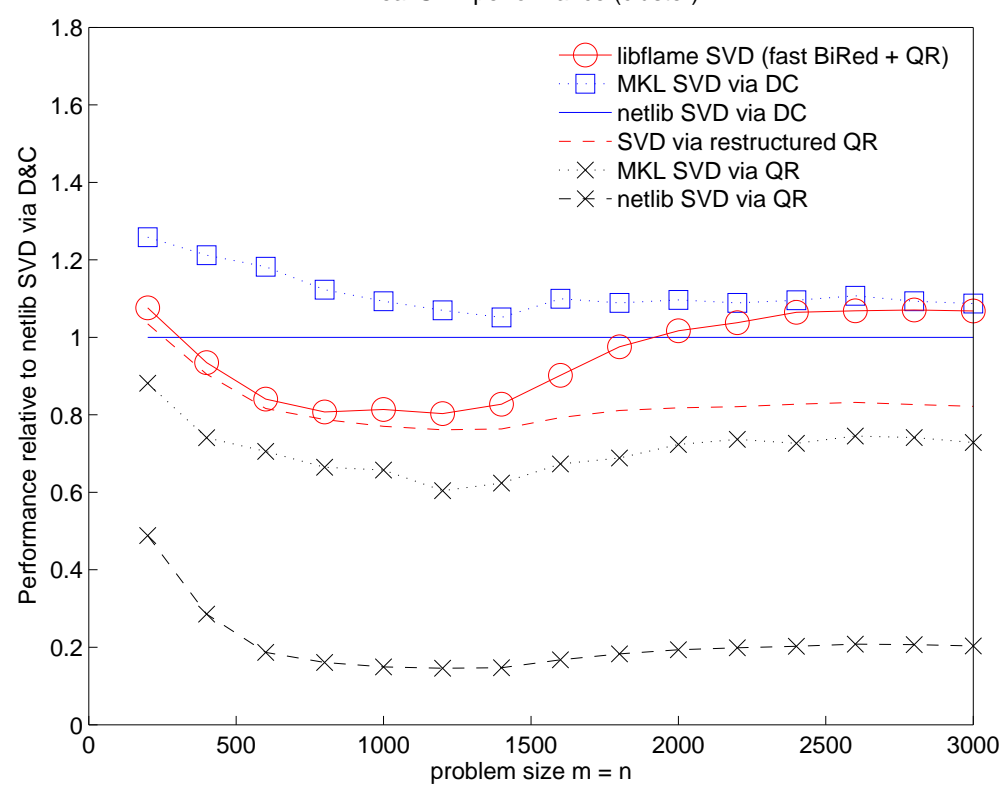

Complex SVD performance (cluster)

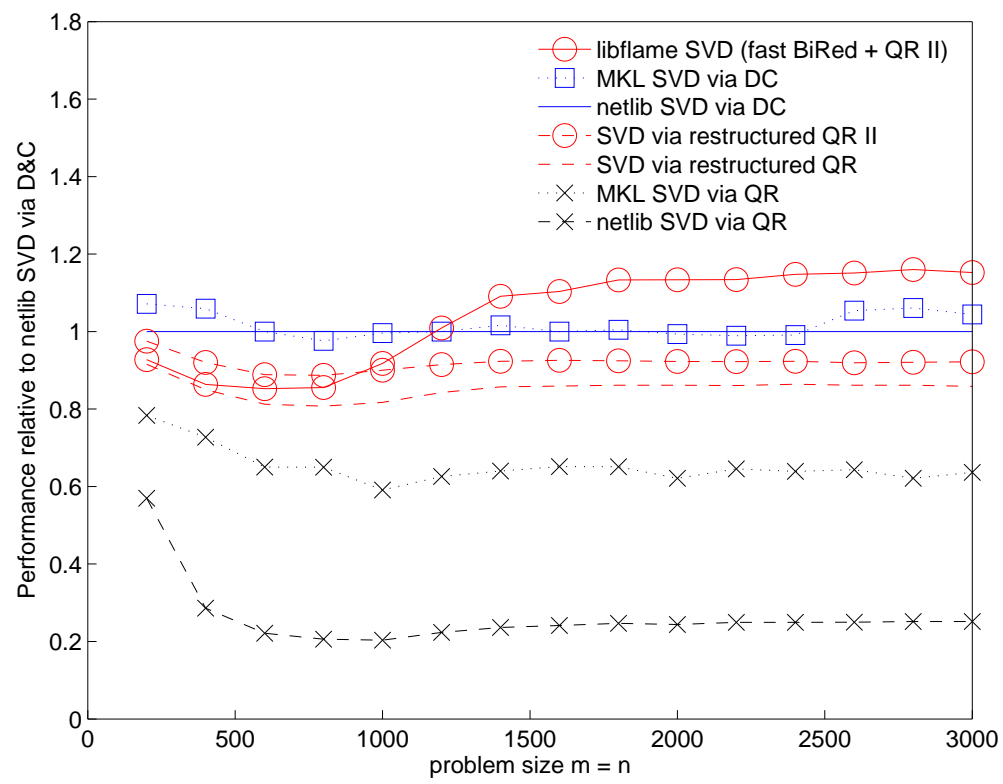

Fig. 29. Performance of implementations relative to netlib SVD via D\&C for real (top) and complex (bottom) matrices generated with clustered distributions of singular values. Here, the problem sizes listed are $m=n$. In the case of SVD via restructured QR, matrices were reduced to bidiagonal form using an implementation that exhibits a performance signature nearly identical to that of dgebrd/zgebrd. As explained in Section 9.4, results for "libflame SVD (fast BiRed + QR)" (top) and "libflame SVD (fast BiRed + QR II)" (bottom) combine the higher-performing bidiagonal reduction presented in [Van Zee et al. 2012] with the restructured QR algorithm. 
Real SVD performance (geometric)

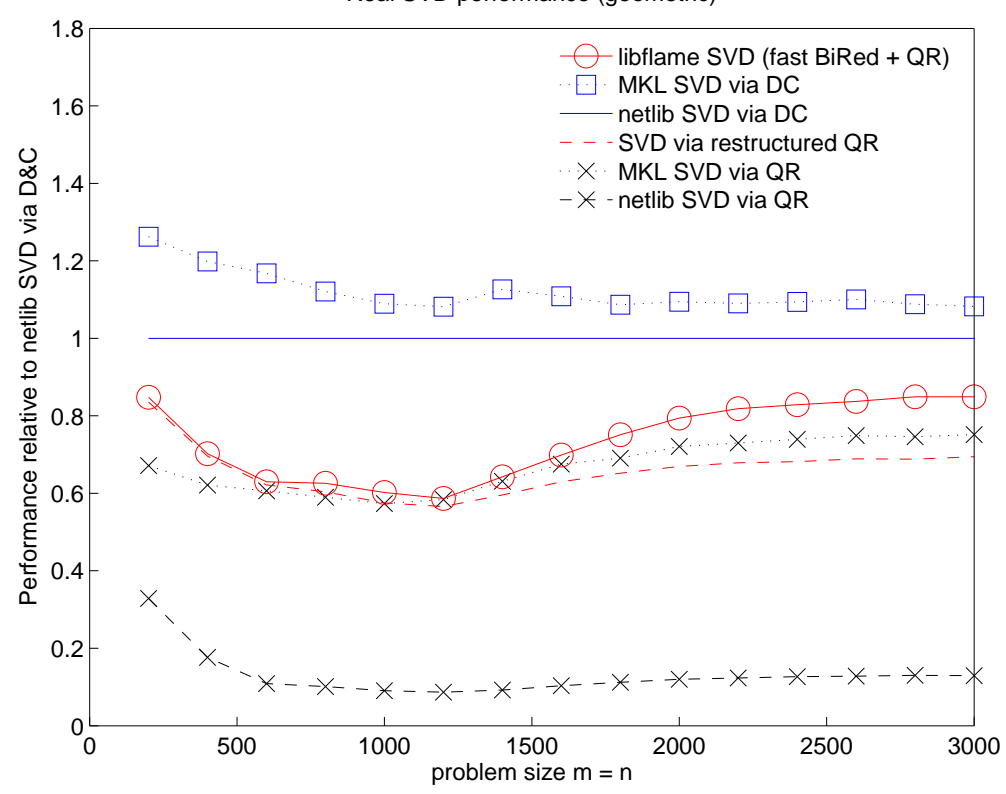

Complex SVD performance (geometric)

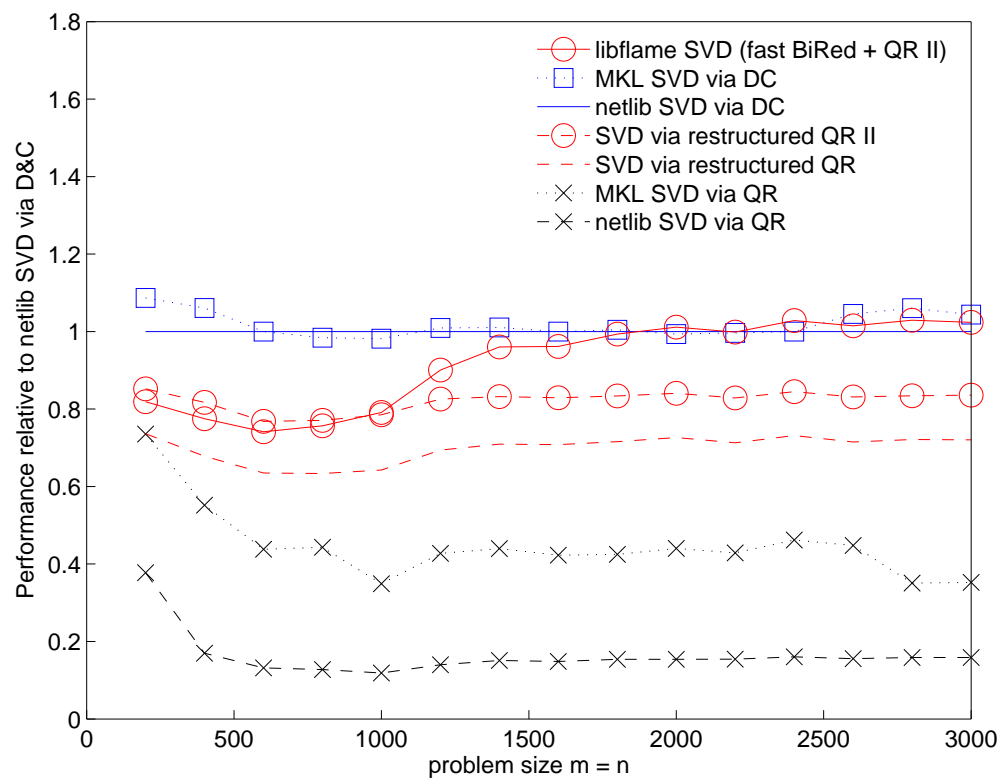

Fig. 30. Performance of implementations relative to netlib SVD via D\&C for real (top) and complex (bottom) matrices generated with geometric distributions of singular values. Here, the problem sizes listed are $m=n$. In the case of SVD via restructured QR, matrices were reduced to bidiagonal form using an implementation that exhibits a performance signature nearly identical to that of dgebrd/zgebrd. As explained in Section 9.4, results for "libflame SVD (fast BiRed + QR)" (top) and "libflame SVD (fast BiRed + QR II)" (bottom) combine the higher-performing bidiagonal reduction presented in [Van Zee et al. 2012] with the restructured QR algorithm. 
Real SVD performance (inverse)

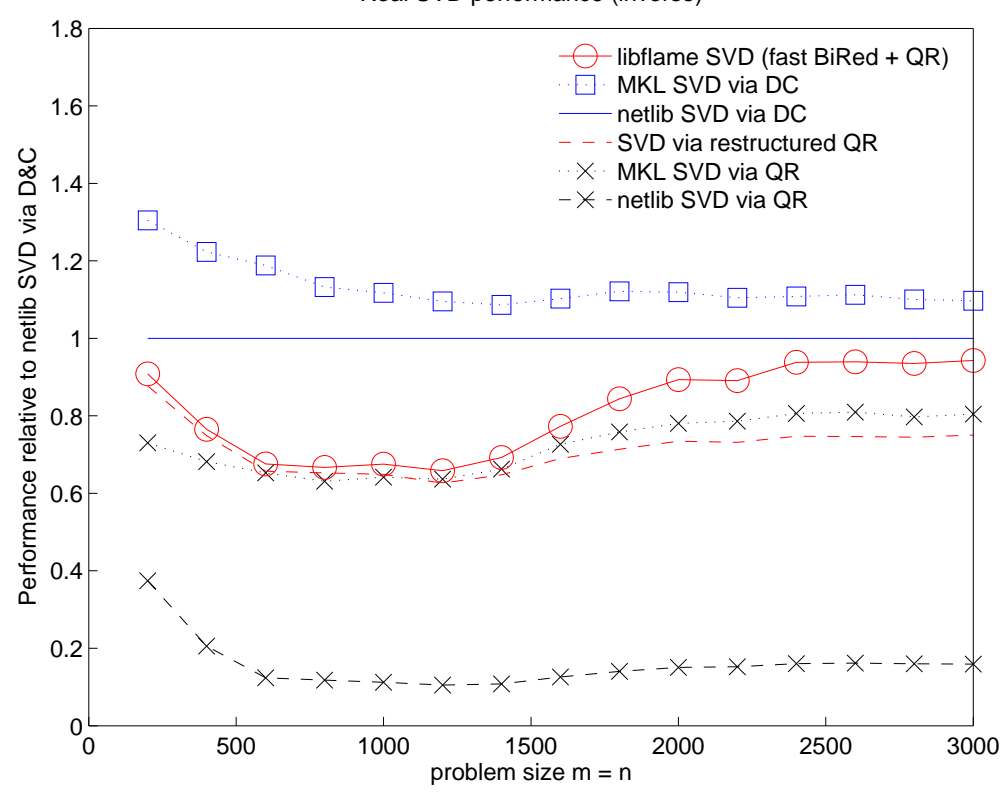

Complex SVD performance (inverse)

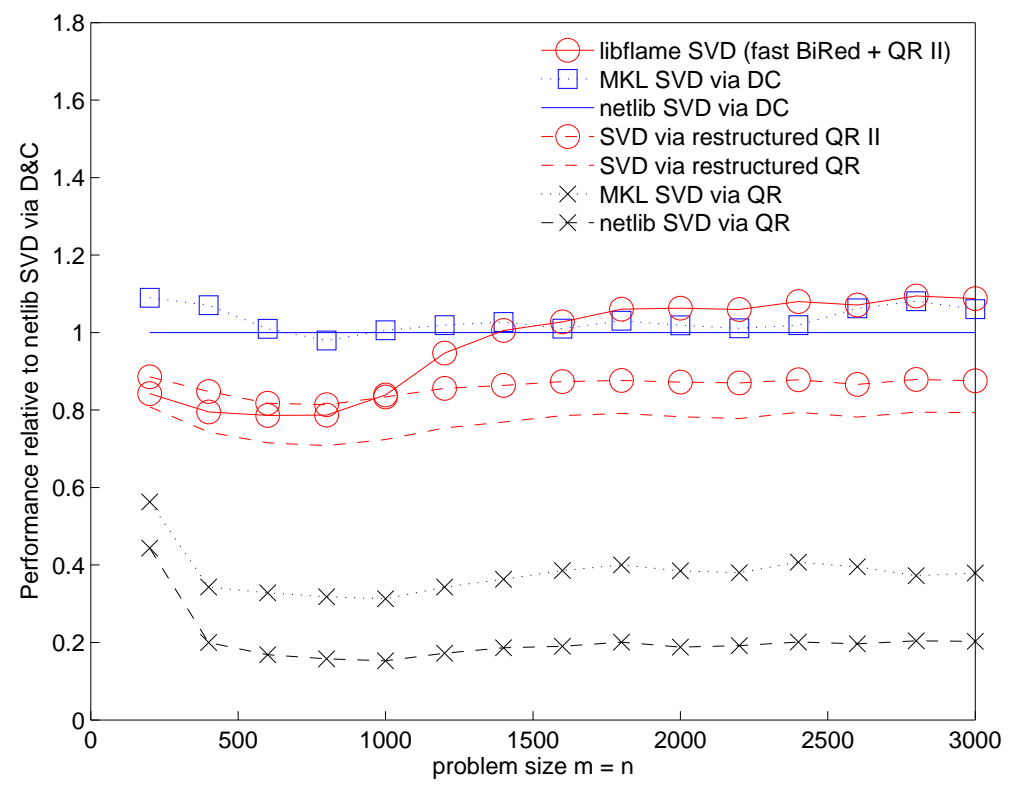

Fig. 31. Performance of implementations relative to netlib SVD via D\&C for real (top) and complex (bottom) matrices generated with inverse distributions of singular values. Here, the problem sizes listed are $m=n$. In the case of SVD via restructured QR, matrices were reduced to bidiagonal form using an implementation that exhibits a performance signature nearly identical to that of dgebrd/zgebrd. As explained in Section 9.4, results for "libflame SVD (fast BiRed + QR)" (top) and "libflame SVD (fast BiRed + QR II)" (bottom) combine the higher-performing bidiagonal reduction presented in [Van Zee et al. 2012] with the restructured QR algorithm. 

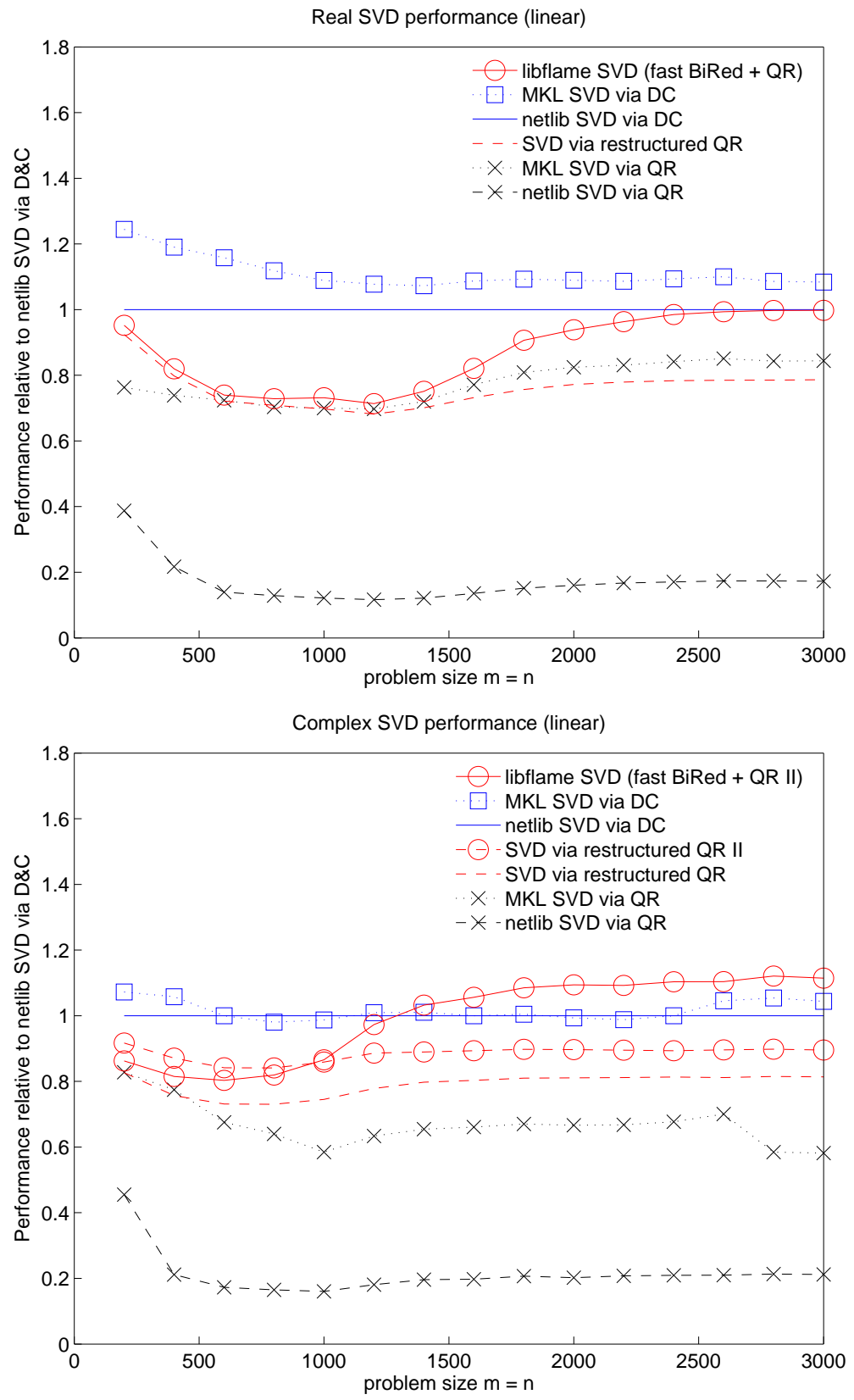

Fig. 32. Performance of implementations relative to netlib SVD via D\&C for real (top) and complex (bottom) matrices generated with linear distributions of singular values. Here, the problem sizes listed are $m=n$. In the case of SVD via restructured QR, matrices were reduced to bidiagonal form using an implementation that exhibits a performance signature nearly identical to that of dgebrd/zgebrd. As explained in Section 9.4, results for "libflame SVD (fast BiRed + QR)" (top) and "libflame SVD (fast BiRed + QR II)" (bottom) combine the higher-performing bidiagonal reduction presented in [Van Zee et al. 2012] with the restructured QR algorithm. 
Real SVD performance (logarithmic)

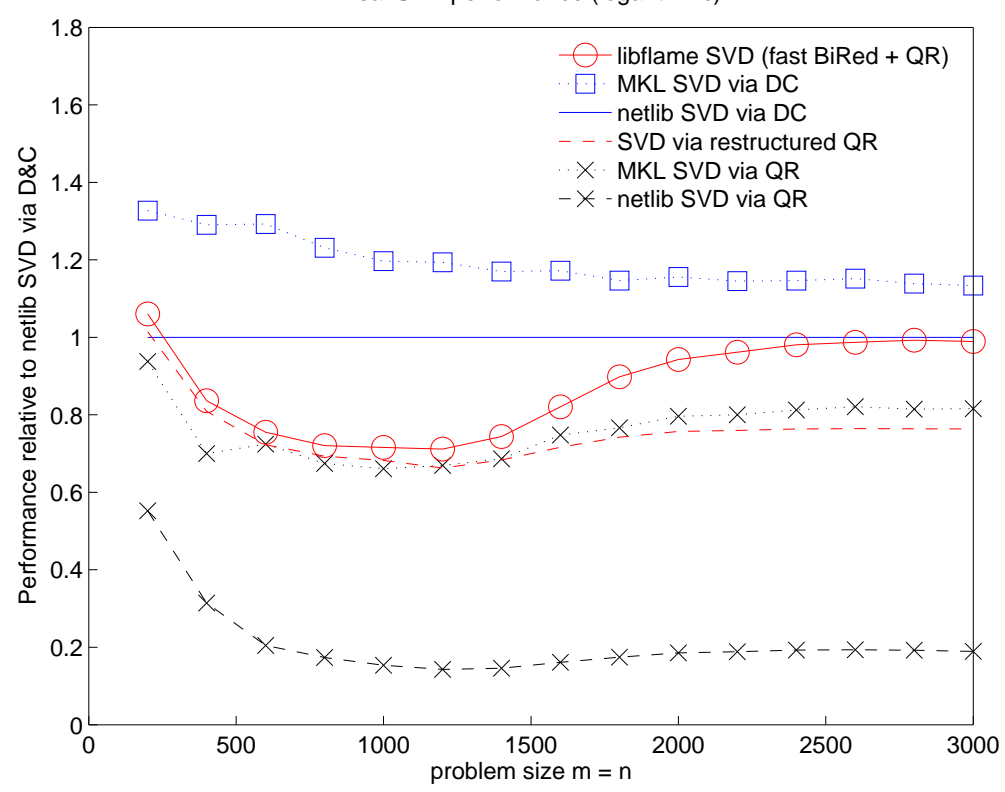

Complex SVD performance (logarithmic)

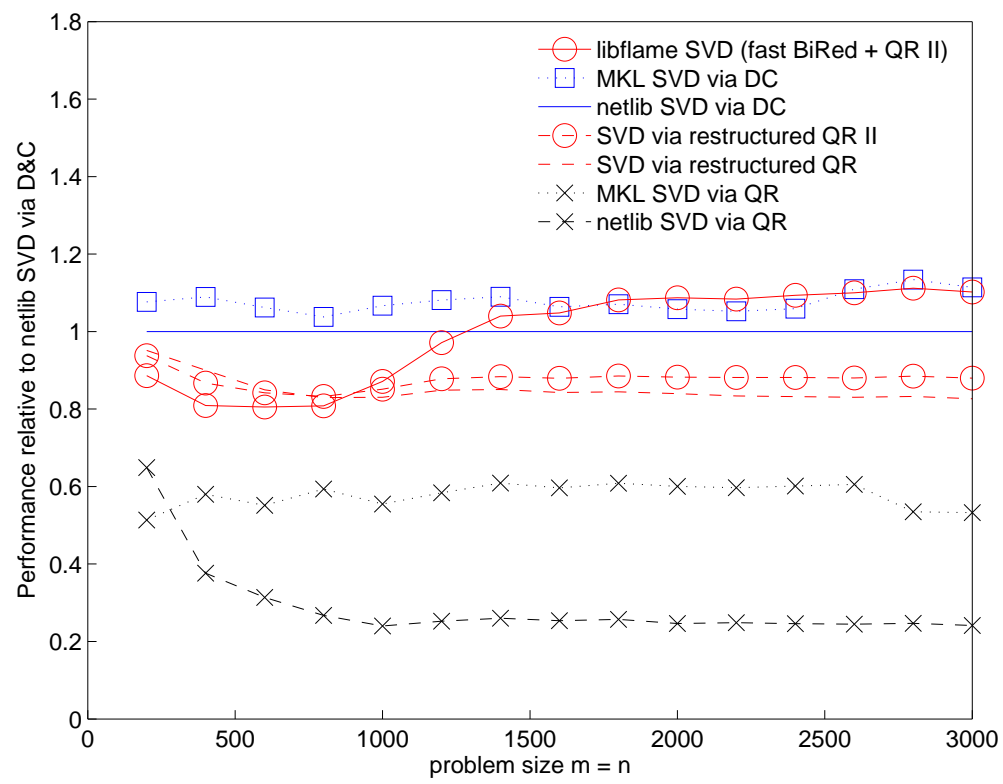

Fig. 33. Performance of implementations relative to netlib SVD via D\&C for real (top) and complex (bottom) matrices generated with logarithmic distributions of singular values. Here, the problem sizes listed are $m=n$. In the case of SVD via restructured QR, matrices were reduced to bidiagonal form using an implementation that exhibits a performance signature nearly identical to that of dgebrd/zgebrd. As explained in Section 9.4, results for "libflame SVD (fast BiRed + QR)" (top) and "libflame SVD (fast BiRed + QR II)" (bottom) combine the higher-performing bidiagonal reduction presented in [Van Zee et al. 2012] with the restructured QR algorithm. 
Real SVD performance (random)

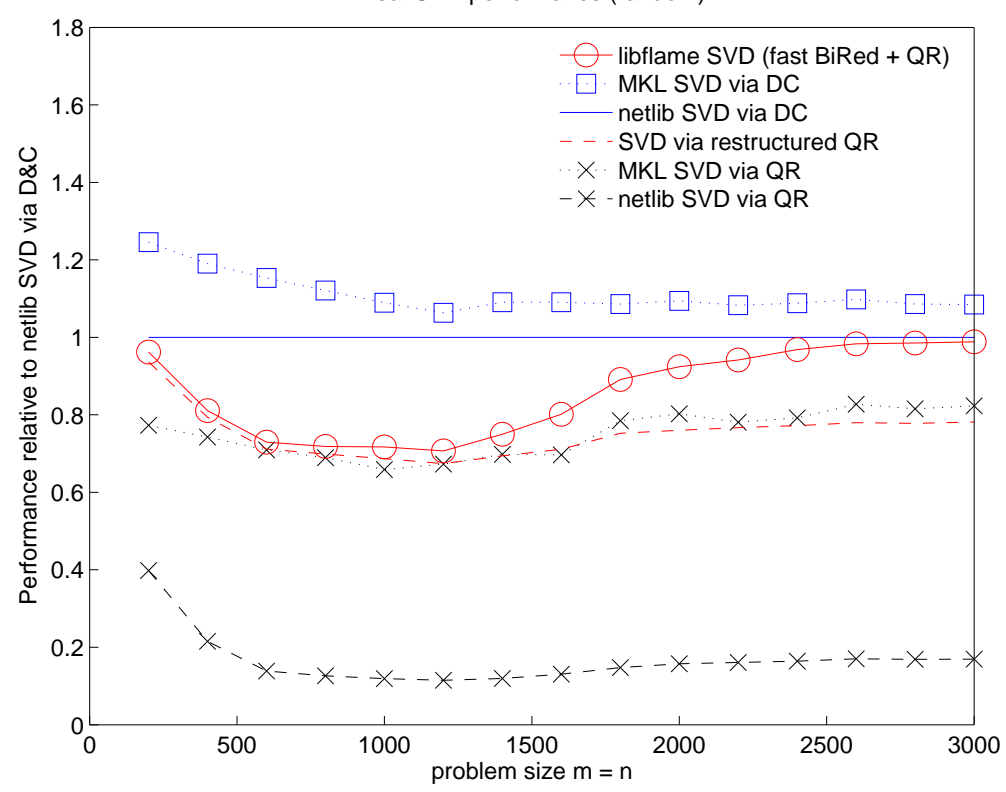

Complex SVD performance (random)

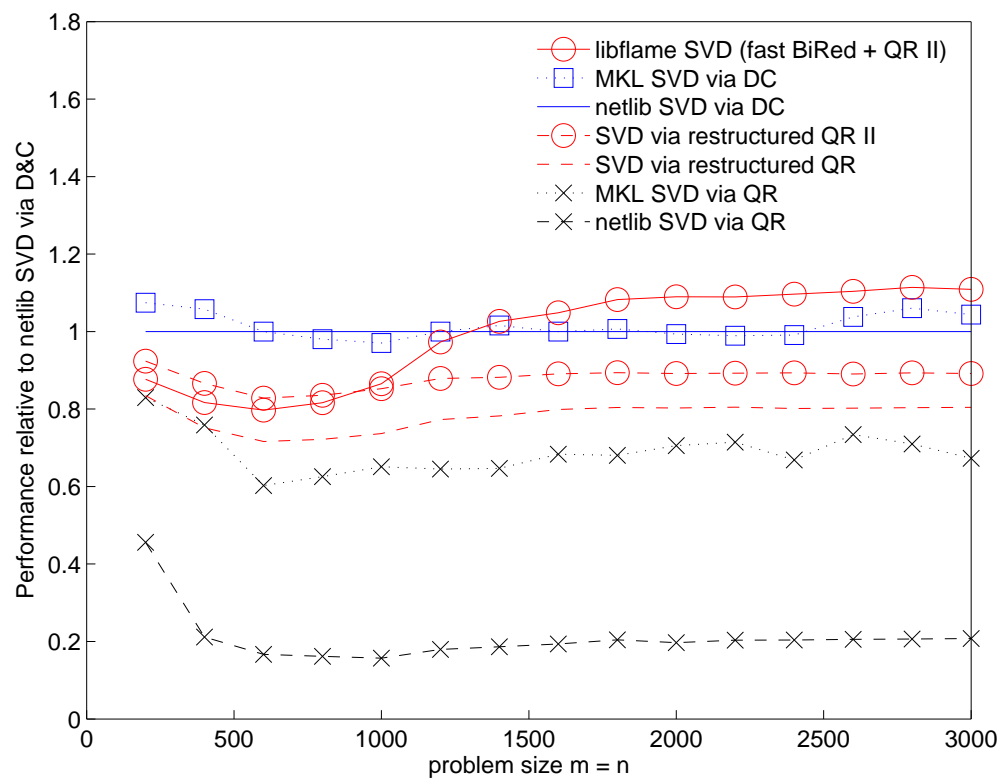

Fig. 34. Performance of implementations relative to netlib SVD via D\&C for real (top) and complex (bottom) matrices generated with random distributions of singular values. Here, the problem sizes listed are $m=n$. In the case of SVD via restructured QR, matrices were reduced to bidiagonal form using an implementation that exhibits a performance signature nearly identical to that of dgebrd/zgebrd. As explained in Section 9.4, results for "libflame SVD (fast BiRed + QR)" (top) and "libflame SVD (fast BiRed + QR II)" (bottom) combine the higher-performing bidiagonal reduction presented in [Van Zee et al. 2012] with the restructured QR algorithm. 